\title{
A Novel Energy-Efficient, Static Scenario-Oriented Routing Method of Wireless Sensor Network Based on Edge Computing
}

\author{
Gang Liu $\mathbb{D}^{1},{ }^{1}$ Zhaobin Liu $\mathbb{D},{ }^{1}$ Victor S. Sheng, ${ }^{2}$ Liang Zhang, ${ }^{1}$ and Yuanfeng Yang ${ }^{1}$ \\ ${ }^{1}$ School of Computer Engineering, Suzhou Vocational University, Suzhou 215104, China \\ ${ }^{2}$ Department of Computer Science, Texas Tech University, Texas 79409, USA \\ Correspondence should be addressed to Zhaobin Liu; lzb_jssvc@163.com
}

Received 11 February 2020; Revised 7 September 2021; Accepted 20 December 2021; Published 15 January 2022

Academic Editor: Yejun He

Copyright (c) 2022 Gang Liu et al. This is an open access article distributed under the Creative Commons Attribution License, which permits unrestricted use, distribution, and reproduction in any medium, provided the original work is properly cited.

\begin{abstract}
In wireless sensor network (WSN), the energy of sensor nodes is limited. Designing efficient routing method for reducing energy consumption and extending the WSN's lifetime is important. This paper proposes a novel energy-efficient, static scenario-oriented routing method of WSN based on edge computing named the NEER, in which WSN is divided into several areas according to the coverage of gateway (or base station), and each of the areas is regarded as an edge area network (EAN). Each edge area network is abstracted into a weighted undirected graph model combined with the residual energy of the sensor nodes. The base station (or a gateway) calculates the optimal energy consumption path for all sensor nodes within its coverage, and the nodes then perform data transmission through their suggested optimal paths. The proposed method is verified by the simulations, and the results show that the proposed method may consume about 37\% less energy compared with the conventional WSN routing protocol and can also effectively extend the lifetime of WSN.
\end{abstract}

\section{Introduction}

In recent years, the edge computing paradigm has gained considerable popularity in academic and industrial circles. It serves as a key enabler for many future technologies, such as $5 \mathrm{G}$ [1], augmented reality, and the Internet of Things (IoT) [2]. Wireless sensor networks (WSNs), as a key technology of the Internet of Things [3], have achieved great success in the fields of environmental monitoring [4], smart cities [5], medical care [6,7], target tracking [8], and behavioral monitoring [9]. Thus, WSNs have aroused widespread attention in academia and industry.

A WSN is composed of a large number of static or movable microsensor nodes deployed in the monitoring area. The data monitored by these sensor nodes are transmitted to other sensor nodes, hop by hop, through wireless communication. During transmission, the monitored data can be processed by different nodes and reach the base station (or a gateway) through a multihop route. The monitored data collaboratively perceive, collect, process, and transmit information about objects within the network coverage area, and this information is then sent to the network manager through the Internet or by a satellite, as demonstrated in Figure 1. By seeking an optimal route between the source and destination nodes, routing technologies in a WSN efficiently forward data packets to the destination node through multiple hops, realizing a multihop path. Some general characteristics of WSNs are large scale, self-organization, limited resources, and complex environment.

Since the processing, storage, and communication capabilities of an individual sensor node are relatively weak and since their battery supply is limited, determining how to save energy and prolong the life-cycle of the entire network in the routing process is a crucial issue in WSNs. The existing routing protocols currently have the following problems:

(1) Routing is distributed, and all nodes in a WSN need to implement a routing algorithm, which increases the costs of calculation, storage, and communication

(2) The energy consumption and residual energy of the WSN nodes are not considered in the routing calculation 


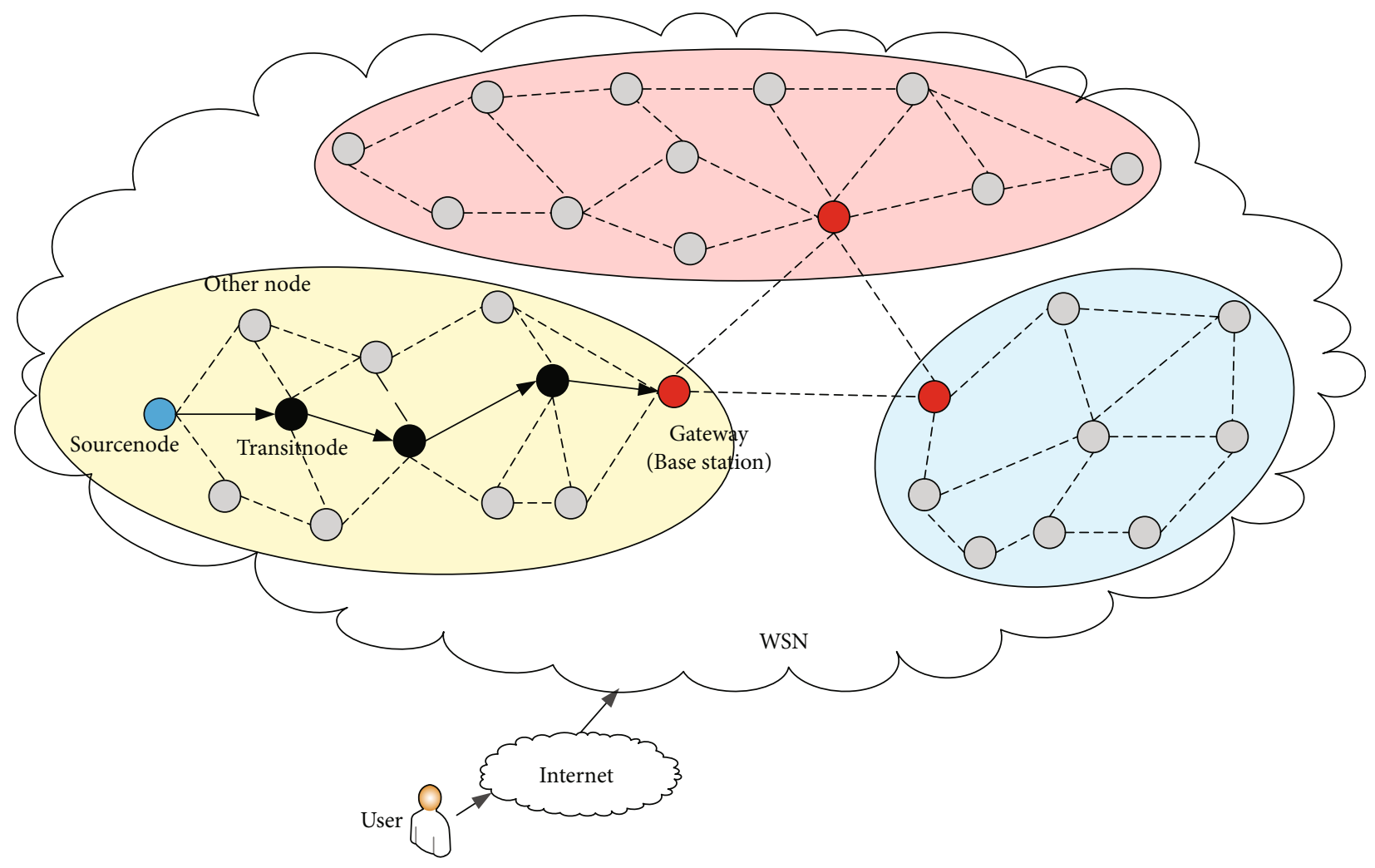

FIgURE 1: WSN structure.

(3) Nodes need to store a large amount of routing information

(4) Routing is completely centralized, which causes high latency

In order to solve the above problems, this paper proposes a novel energy-efficient, static scenario-oriented routing method of WSNs based on edge computing named the NEER. The proposed method is applicable to the scenario where sensor nodes are fixed in a sensor network. Based on the idea of edge computing, a WSN is divided into several edge area networks according to the coverage of the base station (or gateway). The calculation of the residual energy is carried out at the end node, and data routing is performed on the edge area network. The entire routing process of the edge area network is as follows.

First, a base station (or gateway) node acquires the topological information of an edge area network. Second, with the guarantee of network connectivity, the base station (or gateway) calculates an optimal routing path of all sensor nodes of the edge area network to the base station (or gateway) with the purpose of minimizing the energy consumption in a centralized way. Next, the base station (or gateway) node notifies each sensor node about its optimal paths. Finally, the perceived data are routed to the base station (or gateway) by a multihop route, which represents the optimal route. Data transmission between the edge area networks is performed through base stations (or gateways). Because the data transmission of each of the edge area networks in a WSN ensures the minimization of energy consumption, the proposed method achieves the purpose of saving energy, lowering node disturbances, and prolonging the life cycle of the whole WSN.

In this paper, the Contiki/Cooja simulation platform is used to verify the proposed NEER method from the aspects of network lifetime and network energy consumption for different scenarios. The experimental results show that compared with the traditional RPL (IPv6 routing protocol for low-power and lossy networks) protocol $[10,11]$, the proposed method can significantly extend the WSN lifetime by at least $30 \%$ and reduced the energy consumption by approximately $37 \%$.

The main contributions of this work can be summarized as follows:

(1) The proposed method breaks the idea of completely distributed or centralized routing. A WSN is divided into several edge networks based on the edge computing idea. Each edge network adopts centralized routing internally, so the nodes do not need to implement a routing algorithm or save routing information, which not only saves computing cost and storage resources but also reduces the delay

(2) The proposed routing method can select an optimal path to effectively reduce the network energy consumption and extend the network life by fully considering the residual energy of nodes

(3) The proposed method collects the data of all nodes on the path by one-time routing, so the packet 


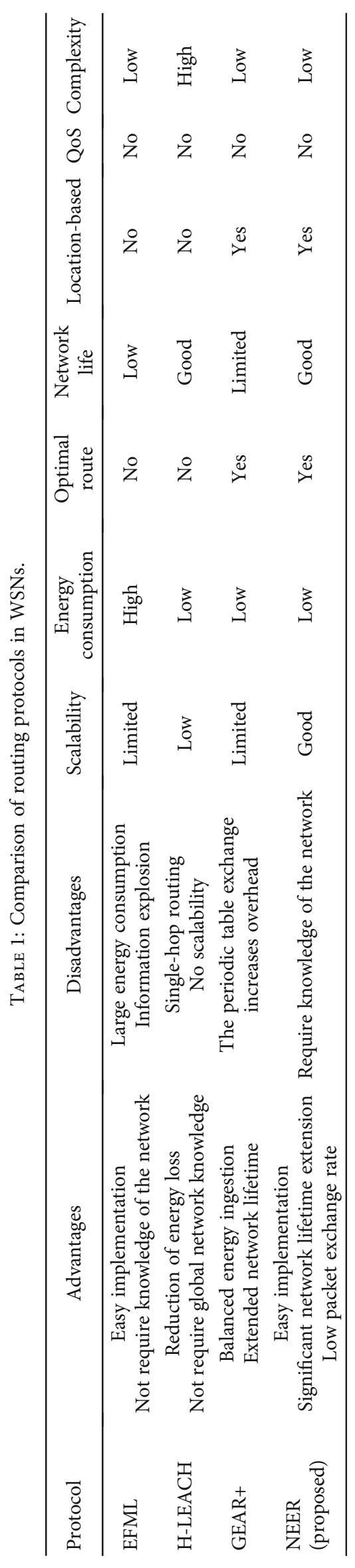


exchange rate is low, and the communication overhead is reduced

The rest of the paper is organized as follows. The related work is described in Section 2. The topological model and energy consumption model related to the NEER are discussed in Section 3. In Section 4, the proposed method and its detailed analysis are presented. In Section 5, the simulation and experimental results are discussed, and the proposed method is compared with the RPL routing protocol. Finally, the conclusions are given in Section 5.

\section{Related Work}

In past years, research on WSN energy-efficient routing methods has achieved significant progress. Currently, the main routing methods are network structure-based routing, communication model-based routing, topology-based routing, and reliability routing.

The energy-efficient, flooding with minimum latency, plane routing algorithm (EFML) proposed by Song et al. [12] belongs to the class of network structure-based routing algorithms. This algorithm first constructs the shortest-path tree with the smallest delay from the sink node to other nodes and then establishes the minimum spanning tree with a given time delay. However, this algorithm has problems related to the information explosion and overlap.

Razaque et al. proposed the hybrid-LEACH (H-LEACH) protocol [13]. To face the energy hole problem of LEACH, the H-LEACH considers the maximum energy of a node, its remaining energy, the energy required for data transmission, and the probability of energy usage and implements a threshold value. The nodes below the threshold are considered dead. Finally, the network lifetime is extended.

To improve the WSN geographic routing protocol and solve the problems of energy wasting and routing hole in data transmission, a new geographic routing protocol GEAR+ was proposed by Yanqin [14]. By making nodes acquire information on two or more adjacent nodes' locations, the probability of routing hole was reduced. Necessary aggregation of data collection on the nodes was carried out, and meanwhile, the states of the nodes were changed appropriately to avoid unnecessary energy consumption.

The quality-of-service-based routing protocols represent reliable routing methods, which are aimed at achieving an equilibrium between the energy consumption and data transmission quality to meet the time delay and bandwidth requirements [15].

The above-mentioned protocols differ from the proposed method in many aspects. The method proposed in this paper is designed for static WSN scenarios, which is quite suitable for environment-sensing applications with unattended and infrequent topology changes, such as intelligent agriculture, forest fire risk monitoring, and environmental data acquisition. In order to clearly explain the advantages of the proposed method over the existing methods, the comparison of their main characteristics is given in Table 1.
TABLE 2: Symbols used in the edge area network model and their explanations.

\begin{tabular}{lc}
\hline Symbol & Description \\
\hline$G$ & Graph represented by a triple \\
$V$ & Set of nodes \\
$E$ & Set of edges \\
$W$ & Weight set of edges \\
$i, j, k$ & Sensor node ID \\
$r_{i}$ & Residual energy of a sensor node $i$ \\
$v_{i}$ & A tuple consisting of $i$ and $r_{i}$ \\
$e$ & An edge formed between nodes $v_{i}$ and $v_{j}$, represented by \\
$d(e) / d_{i j}$ & Distance of the edge formed between nodes $v_{i}$ and $v_{j}$ \\
$c(e) / c_{i j}$ & Energy consumption when the perceived data are \\
$w(e)$ & transmitted or received along edge $e$ \\
HELLO & A tuple consisting of $d(e)$ and $c(e)$ \\
RESP & Neighbor discovery message \\
$x$ & Response message of the neighboring node \\
$y$ & $X$-coordinate of a node \\
TABLE & $Y$-coordinate of a node \\
\hline$d(e)$ & One-hop adjacency table
\end{tabular}

$d(e)$ and $d_{i j}$ are two variables that have the same meaning; $c(e)$ and $c_{i j}$ are also two variables that have the same meaning.

In addition to the above-mentioned classical energyefficient routing protocols, the routing protocols with congestion avoidance [16-18], congestion control [19-21], data aggregating, or multipath function have been designed in recent years. These protocols can effectively reduce the energy consumption of multiple data retransmissions. It should be noted that WSNs are vulnerable to various types of attacks. Therefore, confidentiality, authentication, integrity, availability, and freshness are the main security considerations in WSNs. In order to address these challenges, many secure routing protocols were proposed [22-25]. However, the energy consumption and security are contradictory, so a performance balance between them is necessary. With the aim to achieve this balance, the routing protocols based on multiobjective optimization were proposed in [26-28]. Moreover, with the help of machine learning-based algorithms and the use of the operational experience data of WSN, a self-learning routing protocol was proposed in [29-31] to enhance the network's adaptive characteristics.

The emergence of WSNs has had a profound influence on many fields. Environment monitoring, where various environmental parameters such as temperature, humidity, light, and pressure can be monitored, denotes a typical application of WSN. Recently, environmental monitoring has become one of the important applications for real-time monitoring and protection of the physical world in the field of industry and agriculture.

The application of WSNs in environmental monitoring was studied in [32]. In order to realize agricultural modernization and agricultural environmental protection, $\mathrm{Zhu}$ et al. 


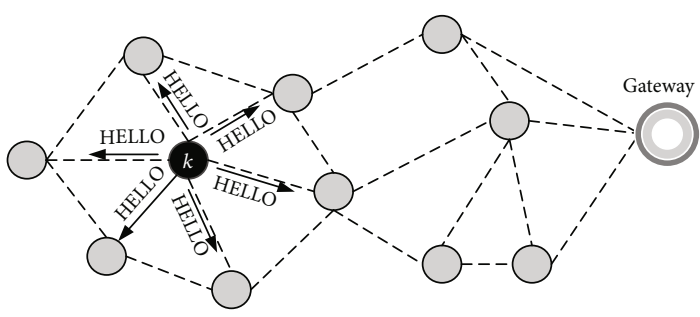

(a)

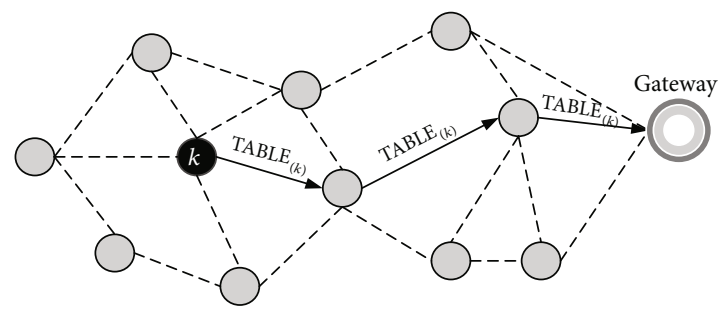

(c)

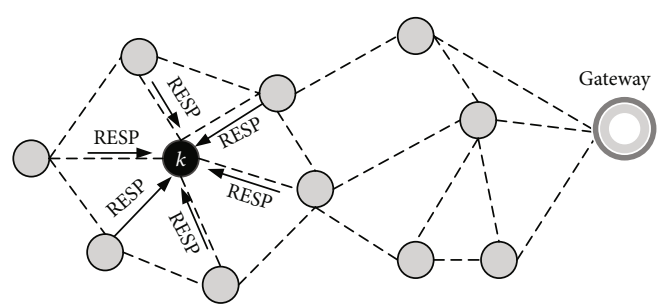

(b)

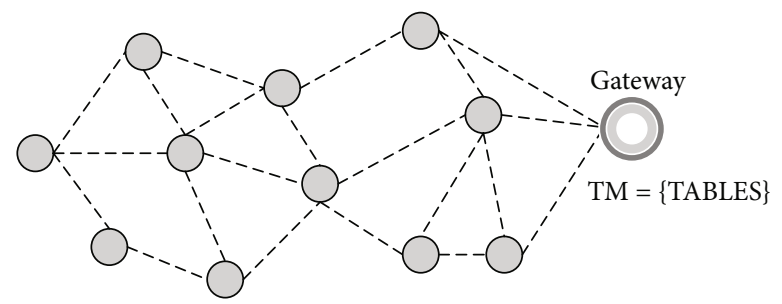

(d)

FIGURE 2: Illustration of the topological model construction: (a) broadcast datagram HELLO; (b) response datagram RESP; (c) passing onehop adjacency table; (d) topology constructed by the base station.

designed a WSN-based agricultural environment monitoring system [33]. In [34], the authors designed an industrial monitoring system based on WSN that could achieve high reliability and low power consumption. As for the medical field, in recent years, with the increase in the population, the medical application market has witnessed explosive growth. In order to improve medical quality, various emerging technologies have been applied to the medical field. Sghaier et al. used the application of WSN technology to monitor the physiological data of patients and track their movement trajectories [35]. In addition to collecting the bioinformation of patients, this system also collects other relevant information, such as environmental temperature and patient image. Lastly, a fall monitoring and accurate positioning system based on the WSN and RFID technology intended for the elderly population was designed in [36].

The mentioned systems consist of a base station, sensing nodes, a routing scheme for the wireless sensor nodes, and a real-time monitoring application that operates from a remote computer and a mobile phone. A routing scheme is a very important part of the data transmission and collection processes in a WSN. Besides, different routing methods have different energy consumptions. Therefore, reducing the energy consumption of sensor nodes to extend the network lifetime is a major concern for an energy-constrained WSN.

\section{Topological Model and Energy Consumption Model}

This section introduces the concepts and models of the proposed NEER method, including the edge area network topological model and its construction algorithm and energy consumption model of sensor nodes.

3.1. Topological Model of Edge Area Network. A WSN that is considered in this study consists of several sensor and base station nodes, which are mutually connected by wireless links. The sensor nodes denote small, static, and resourcelimited devices for environmental monitoring and reporting the acquired data to the base stations. The base stations are static devices with sufficient resources and a longer transmission range compared to the sensor nodes. According to the base station's coverage size, a WSN can be divided into several edge area networks. For the sake of easier understanding of the following explanations of the edge area network model and its parameters, the symbols and their descriptions are given in Table 2.

This paper abstracts an edge area network into a weighted undirected graph model defined as $G=(V, E, W)$, where $V=\left\{v_{1}, v_{2}, \cdots, v_{n}\right\}$ denotes a set of nodes, $E \subseteq V \times V$ is a set of edges in the graph, and $W$ is a weight set of the edges. For all $v \in V, v=\left(i, r_{i}\right)$ is a tuple, where $i$ denotes the node number and $r_{i}$ is the residual energy of node $i$. For all $e \in E, e=\left(v_{i}, v_{j}\right)$ represents the edge formed between nodes $v_{i}$ and $v_{j}$, which is also a tuple. In addition, for all $w$ $(e) \in W, w(e)=(d(e), c(e))$ is a tuple, where $d(e)$ represents the distance of the edge formed between nodes $v_{i}$ and $v_{j}$, and $c(e)$ is energy consumption when the perceived data are transmitted or received along edge $e$. In all cases, $i, j \in\{1$, $2, \cdots, n\}$, and $n$ denotes the total number of nodes.

In order to acquire the topological information of an edge area network, this paper uses the broadcast discovery mechanism. The process of topological model construction, taking a node named $k$ as an example, is illustrated in Figure 2.

In Figure 2(a), HELLO represents the neighbor discovery message sent by a node $k$, and it includes the ID of node $k$ and "hello" text. In Figure 2(b), RESP represents the response message of the neighboring node, including the ID of the neighboring node and its position coordinates $(x, y)$. In Figure 2(c), $\mathrm{TABLE}_{(k)}$ represents the adjacency table of the node $k$, and it includes the ID of each 
1: Set the time period to broadcast a HELLO message as $L$

2: Let the set of all sensor nodes be $V=\left\{v_{1}, v_{2}, \ldots, v_{n}\right\}$

3: While (currentTime $\leq L)\{$

4: $\quad$ For $\forall v_{i} \in V, v_{i}$ periodically broadcasts the HELLO message ( $i$, "hello");

5: $\quad$ if $\left(v_{j}\right.$ receives the HELLO message from $\left.v_{i}, v_{j} \in V, i \neq j\right)\{$

6: $\quad v_{j}$ sends back a response message, $\operatorname{RESP}(j, x, y)$ to $v_{i}$

7: $\quad\}$

8: $\}$

9: for each $\left(v_{k}\right.$ in $\left.V\right)\{$

10: $\quad v_{k}$ generates its one-hop adjacency table denoted as $\operatorname{TABLE}_{(k)}$;

11: $\quad v_{k}$ sends its $\operatorname{TABLE}_{(k)}$ and residual energy $r_{k}$ to the base station (or gateway);

12: $\}$

13: The base station (or gateway) fuses all of the received one-hop adjacency tables to obtain the topological information of the edge area network, which is denoted as TM.

Algorithm 1: Topological model construction algorithm.

TABLE 3: Topological information generated by the base station (or gateway).

\begin{tabular}{lccccccc}
\hline Node ID/residual energy & $1, r_{1}$ & $2, r_{2}$ & $\ldots$ & $\ldots$ & $j, r_{j}$ & $\ldots$ & $n, r_{n}$ \\
\hline $1, r_{1}$ & - & $d_{12}, c_{12}$ & $\ldots$ & $\ldots$ & $d_{1 i}, c_{1 j}$ & $\ldots$ & $d_{1 n}, c_{1 n}$ \\
$2, r_{2}$ & $d_{21}, c_{21}$ & - & $\ldots$ & $\ldots$ & $d_{2 j}, c_{2 j}$ & $\ldots$ & $d_{2 n}, c_{2 n}$ \\
$\ldots$ & $\ldots$ & $\ldots$ & - & $\ldots$ & $\ldots$ & $\ldots$ & $\ldots$ \\
$i, r_{i}$ & $d_{i 1}, c_{i 1}$ & $d_{i 2}, c_{i 2}$ & $\ldots$ & - & $d_{i j}, c_{i j}$ & $\ldots$ & $d_{\text {in }}, c_{\text {in }}$ \\
$\ldots$ & $\ldots$ & $\ldots$ & $\ldots$ & $\ldots$ & $\ldots$ & - & $\ldots$ \\
$n, r_{n}$ & $d_{n 1}, c_{n 1}$ & $d_{n 2}, c_{n 2}$ & $\ldots$ & $\ldots$ & $d_{n j}, c_{n j}$ & $\ldots$ & - \\
\hline
\end{tabular}

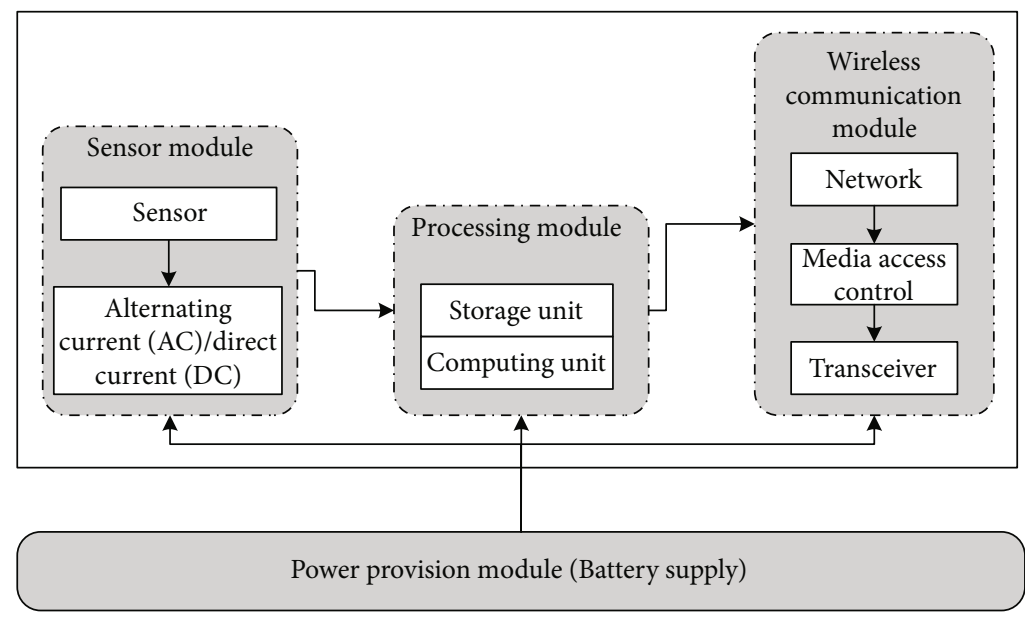

FIgURE 3: Composition of a sensor node.

neighboring node of the node $k$, distance $d$ between the node $k$ and each its neighboring node, and the residual energy $r$ of the node $k$. In Figure 2(d), TM represents the fused topological model information of the adjacency table of all nodes at the base station. The pseudocode of the topological model construction algorithm is provided in Algorithm 1.

The specific steps of Algorithm 1 are as follows:

Step 1. In a predefined period of time (it is assumed that the time is long enough), each sensor node in the edge area network periodically broadcasts a HELLO message.

Step 2. The sensor node receives the HELLO message and sends back an RESP message, which includes its ID and location $(x, y)$.

Step 3. After the broadcasting period of the HELLO message ends, each sensor node generates its one-hop adjacency table. The one-hop adjacency TABLE includes the IDs of 


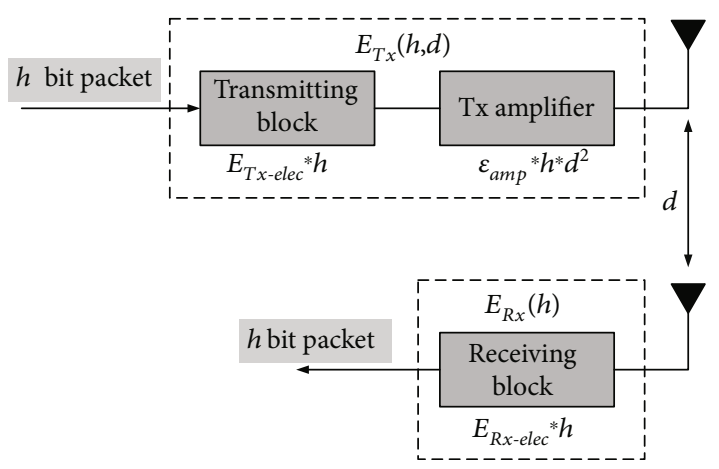

FIgURE 4: The first-order radio model.

TABLE 4: Symbols used in the energy consumption model and their descriptions.

\begin{tabular}{lc}
\hline Symbol & Description \\
\hline$E_{\mathrm{Tx} \text {-elec }}$ & Energy dissipated per bit in the transmitter circuitry \\
$E_{\mathrm{Rx} \text {-elec }}$ & Energy required per bit for successful reception \\
$d$ & Transmission distance \\
$h$ & The number of bits per packet \\
$\varepsilon_{\mathrm{amp}}$ & $\begin{array}{r}\text { Energy consumption of amplifier } \mathrm{Tx} \text {, which is a } \\
\text { constant value of } 100 \mathrm{pJ} / \mathrm{bit} / \mathrm{m}^{2}\end{array}$ \\
$E_{\mathrm{Tx}}(h, d)$ & $\begin{array}{r}\text { Energy consumption when transmitting } h \text {-bit data with } \\
\text { transmission distance } d\end{array}$ \\
$E_{\mathrm{Rx}}(h)$ & Energy consumption when receiving $h$-bit data \\
\hline
\end{tabular}

all neighboring nodes and distances $d$ from the neighboring nodes. The distance $d$ can be calculated by the Euclidean distance formula using the location coordinates of the sensor nodes $v_{i}$ and $v_{j}$.

Step 4. All sensor nodes transmit their one-hop adjacency tables and residual energy information to the base station (or gateway).

Step 5. The base station fuses all of the received one-hop adjacency tables to obtain the topological information of the edge area network.

It can be seen from the pseudocode of Algorithm 1 that sending HELLO message is the core of the algorithm. The lines 4-7 need to be executed $n *(n-1) / 2$ times, and the lines 10-11 need to be executed $n$ times. In the worst case, the complexity of the topological model construction algorithm is $O\left(n^{2}\right)$, where $n$ denotes the number of sensor nodes.

The topological information generated after the base station fuses the adjacency tables of all the nodes is given in Table 3.

In Table $3, n$ denotes the total number of sensor nodes, $i, j$ represent node's ID, $r_{i}$ denotes the residual energy of a node $i, d_{i j}$ is the distance between nodes $i$ and $j$, and $c_{i j}$ is the energy consumed by node $i$ when transmitting data to node $j$.

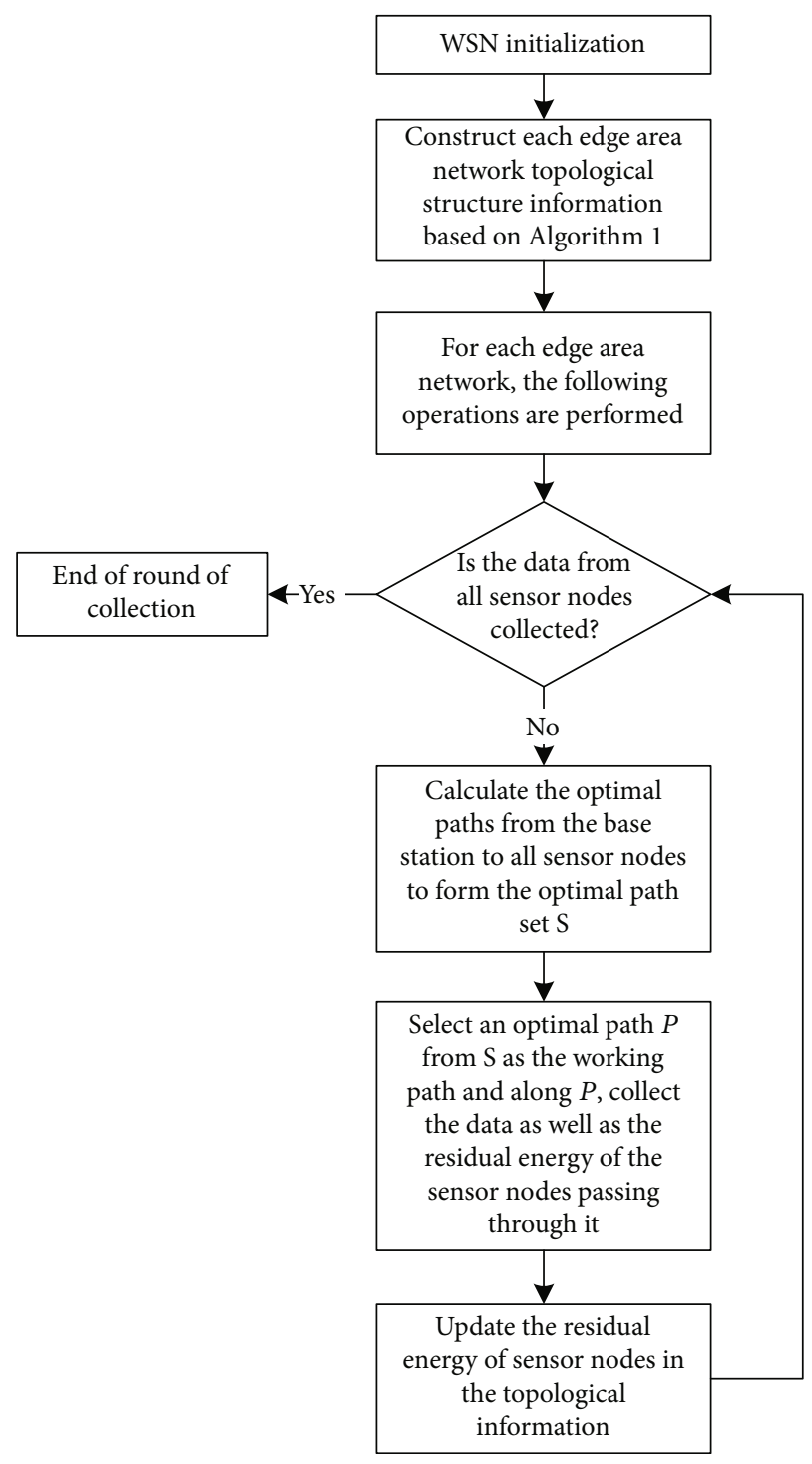

FIGURE 5: Flowchart of WSN data collection.

TABLE 5: Symbols used in the NEER method and their descriptions.

\begin{tabular}{lc}
\hline Symbol & Description \\
\hline$P$ & A path from a sensor node $v$ to the base station \\
$v_{i}$ & Sensor node $i$ \\
base $/ v_{0}$ & Base station \\
$E_{\text {start }}$ & Energy consumption of the first sensor node \\
$E_{\text {others }}$ & Energy consumption of any sensor node other than the \\
$d$ & first sensor node \\
$h$ & Transmission distance \\
$E_{\mathrm{Tx}}(h, d)$ & The number of bits per packet \\
$E_{\mathrm{Rx}}(h)$ & Energy consumption when receiving $h$-bit data \\
$V$ & transmission distance $d$ \\
$S$ & Set of nodes \\
$T$ & Set of not-traversed nodes
\end{tabular}




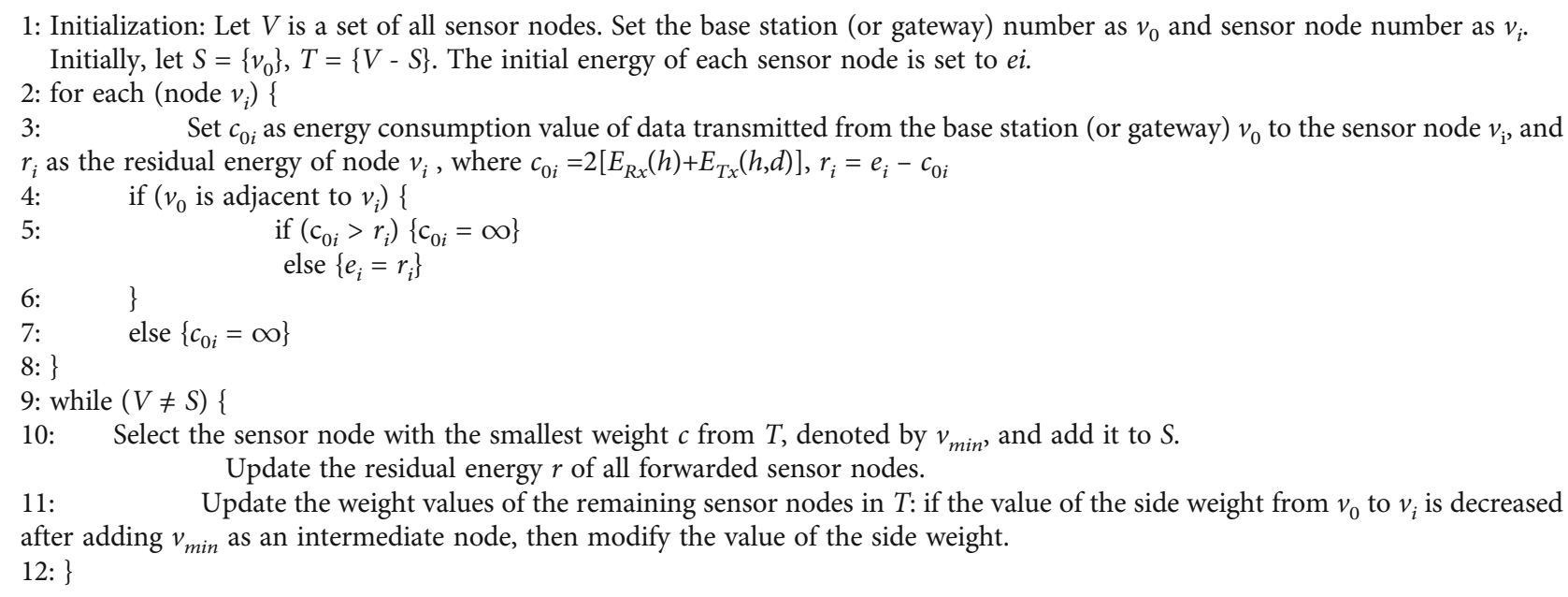

Algorithm 2: Algorithm for optimal path calculation of edge area network.

3.2. Energy Consumption Model. As a key element of a WSN, a sensor node is composed of four parts [37]: a sensor module, a processing module, a wireless communication module, and a power provision module (battery supply), as illustrated in Figure 3. In addition to the local information collection and data processing, a sensor node also receives and forwards the data sent from the other nodes in a network.

In this paper, the first-order radio model [16] is used for energy consumption calculation during data transmission, as shown in Figure 4. The symbols used in the energy consumption model and their descriptions are given in Table 4.

As shown in Figure 4, the energy is mainly consumed by the transmitting module (transmitting block), amplifying module ( $\mathrm{Tx}$ amplifier), and receiving module (receiving block). The energy consumption of the transmitting and receiving modules is the same (i.e., $E_{\mathrm{Tx}-\mathrm{elec}}=E_{\mathrm{Rx}-\mathrm{elec}}=E_{\mathrm{elec}}$ $=50 \mathrm{~nJ} / \mathrm{bit})$ and the energy consumption of Tx amplifier is $\varepsilon_{\text {amp }}=100 \mathrm{pJ} / \mathrm{bit} / \mathrm{m}^{2}$.

The energy consumptions of data transmission and receiving are, respectively, expressed as

$$
\begin{aligned}
E_{\mathrm{Tx}}(h, d) & =E_{\text {elec }} \times h+e_{\mathrm{amp}} \times h \times d^{2}, \\
E_{\mathrm{Rx}}(h) & =E_{\text {elec }} \times h,
\end{aligned}
$$

where $h$ denotes the number of bits per packet and $d$ is the transmission distance.

\section{NEER Description and Analysis}

As a WSN is an application-oriented network, sensor nodes with fixed locations have a wide range of application scenarios in the field. One of the most typical applications of fixed sensors is for environmental monitoring. The routing method proposed in this paper is based on the following three assumptions:

(1) All sensor node positions are fixed
(2) Each sensor node can calculate its current residual energy

(3) The real-time requirements are not high

The NEER method and its execution process, calculation of energy consumption, and selection of optimal path are introduced in detail in the following. The validity of the NEER method is analyzed and proved theoretically.

\subsection{NEER Method}

4.1.1. NEER Process. The NEER method includes five main steps: network initialization, edge area network topological model construction, optimal path calculation, perceived data transmission, and the topological model update. The specific steps of the data acquisition process are as follows:

Step 1. Network initialization, which involves dividing the edge area network, specifying a unique ID for each sensor node, assigning the associated edge area network for each sensor node, setting the initial energy of the sensor node as $R$, and the maximum number of bits of data transmission as $h$.

Step 2. Construct the topological structure information of each edge area network using Algorithm 1.

For each edge area network, perform Steps 3-8.

Step 3. Based on the constructed topological information, calculate the optimal path from the base station (or gateway) to all sensor nodes to form an optimal path set.

Step 4. According to the set screening rule, one of the optimal path sets is selected as a working path, and the information on the working path is sent to all sensor nodes on the working path, where it is stored. 


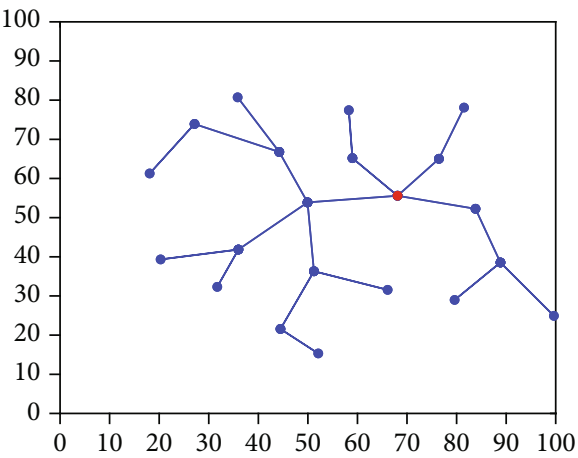

(a)

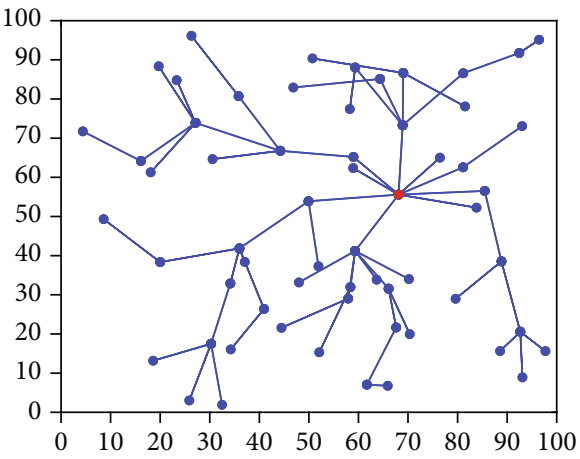

(c)

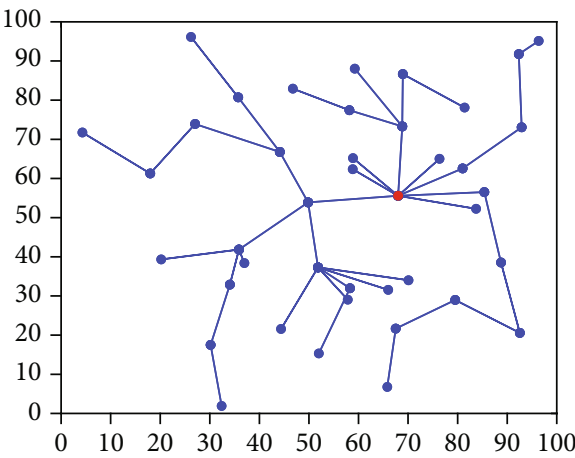

(b)

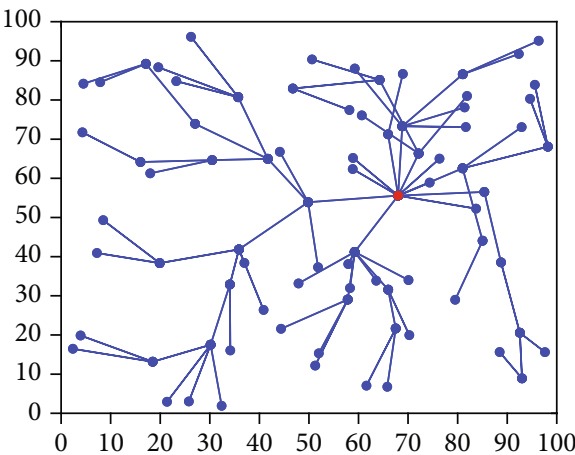

(d)

FIgURE 6: Topological structure of a single-edge area network constructed by the RPL: (a) 20 sensor nodes; (b) 40 sensor nodes; (c) 60 sensor nodes; (d) 80 sensor nodes.

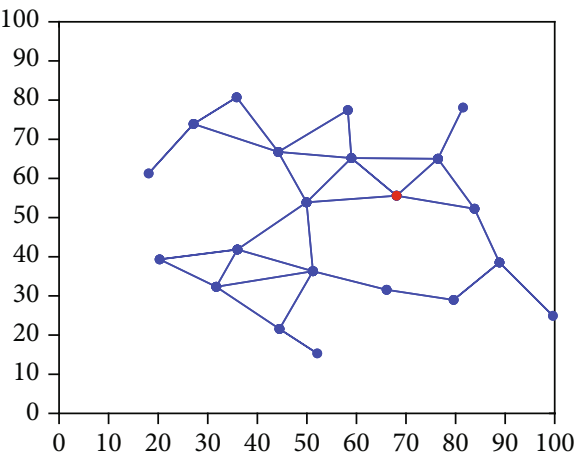

(a)

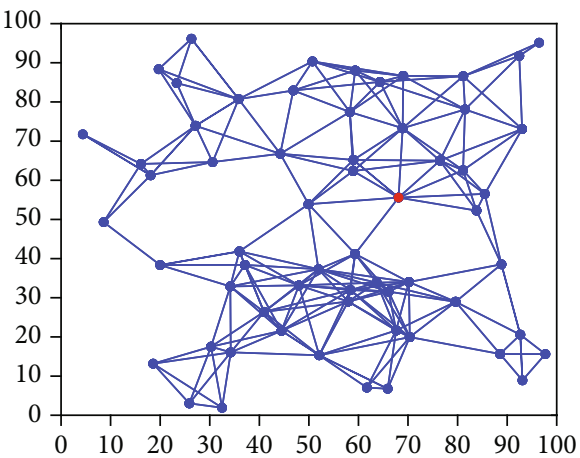

(c)

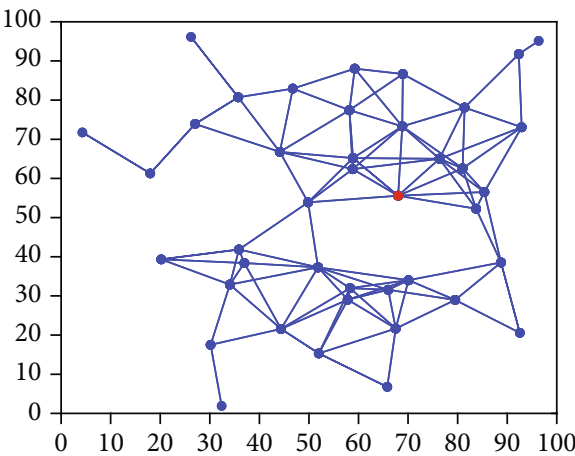

(b)

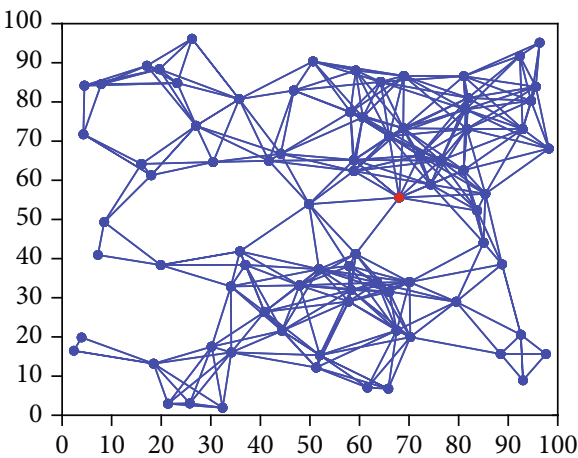

(d)

FIGURE 7: Topological structure of a single-edge area network constructed by the NEER: (a) 20 sensor nodes; (b) 40 sensor nodes; (c) 60 sensor nodes; (d) 80 sensor nodes. 


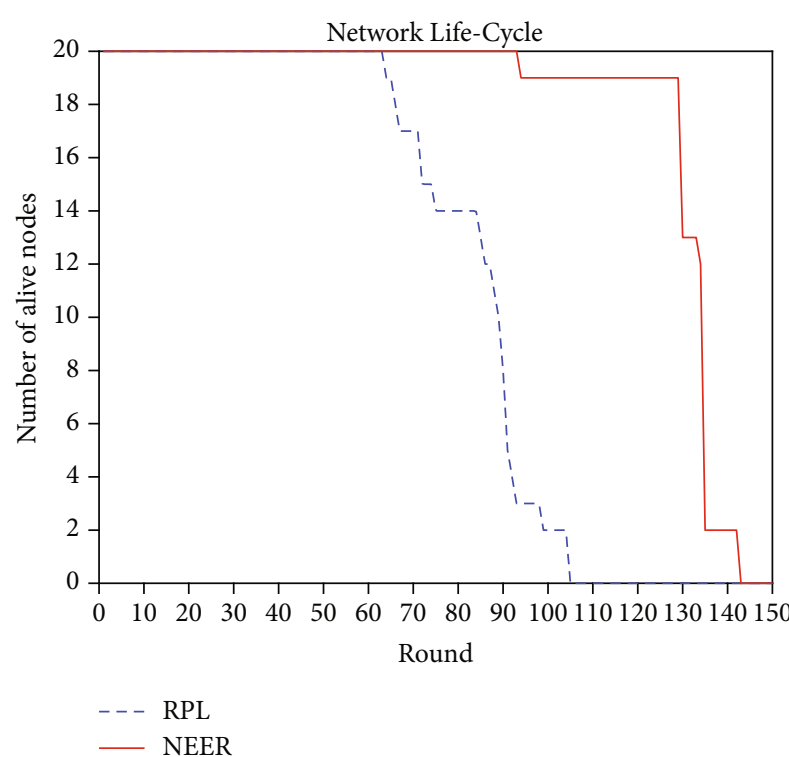

(a)

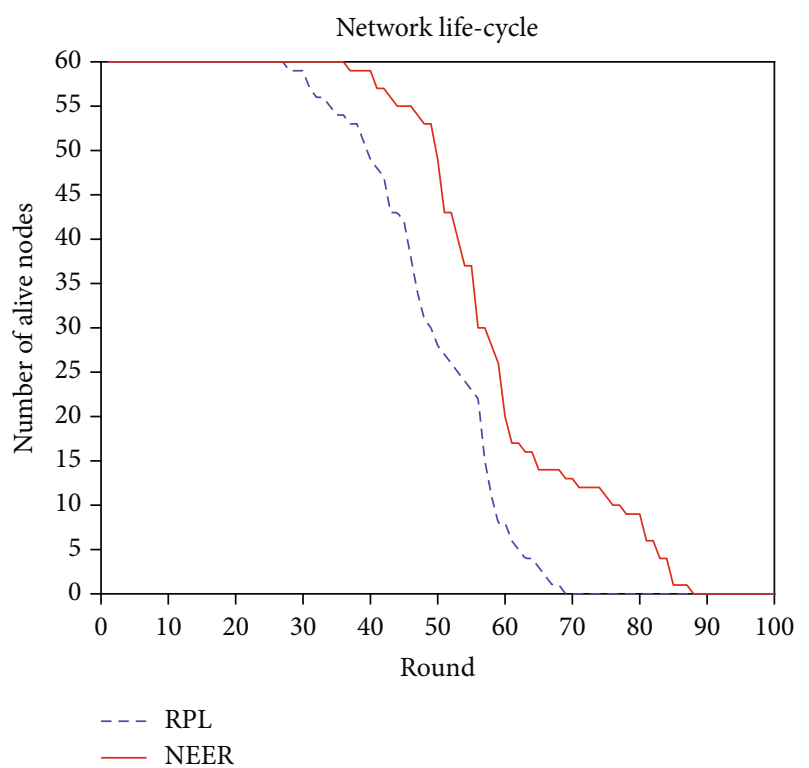

(c)

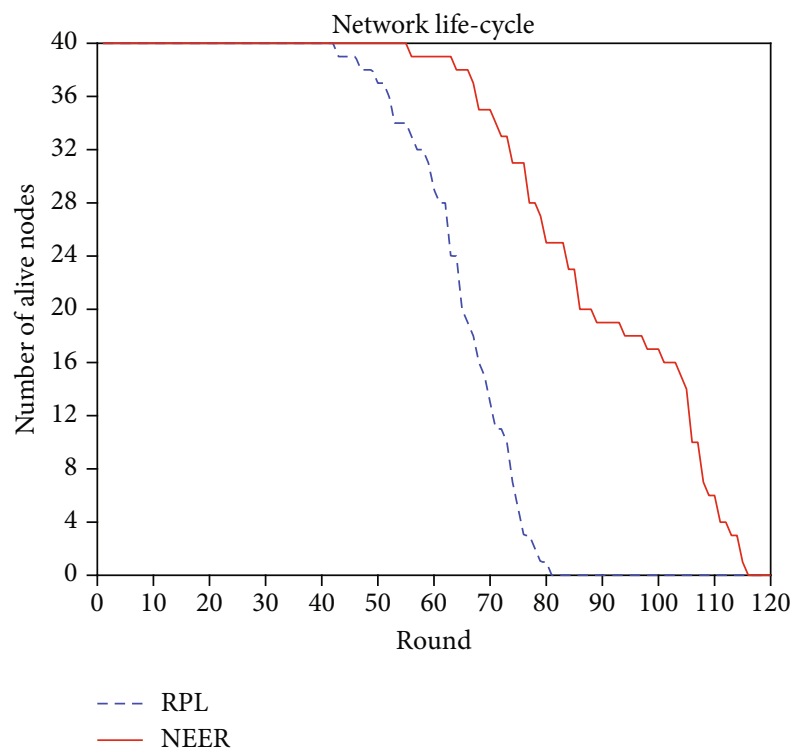

(b)

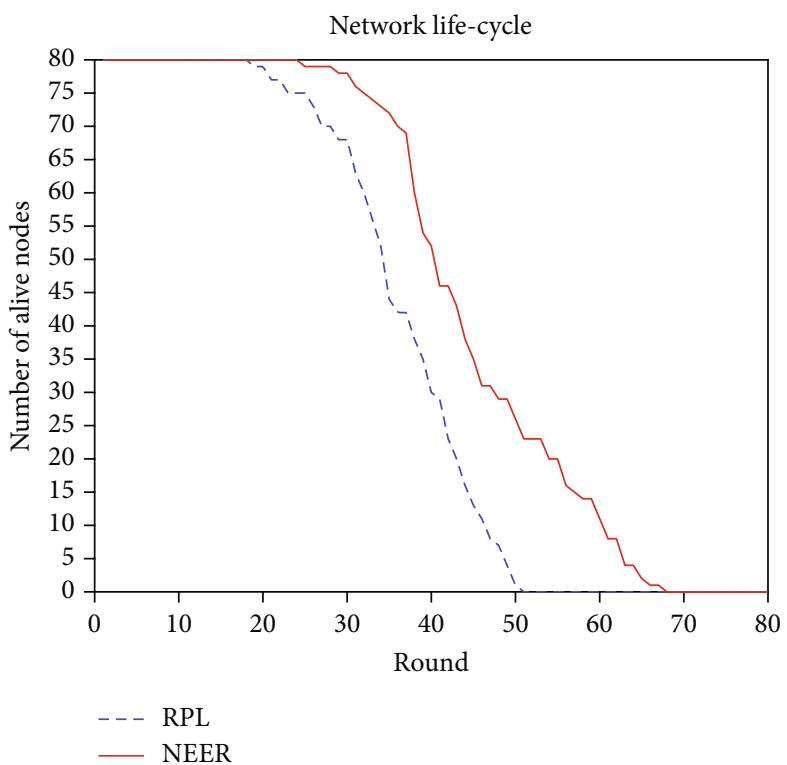

(d)

Figure 8: The network life cycle in a single-edge area network: (a) 20 sensor nodes; (b) 40 sensor nodes; (c) 60 sensor nodes; (d) 80 sensor nodes.

Step 5. The first sensor node on the working path forwards its perceived data and residual energy to the next sensor node on the working path in a hop. The next sensor node fuses the received data with its perceived data and residual energy and forwards it to the following sensor node in a hop, and so on, until the base station (or gateway) receives the data of all sensor nodes on the working path.

Step 6. The base station (or gateway) receives the perceived data, as well as the residual energy of all sensor nodes on the working path. Using the received data, the base station (or gateway) updates the residual energy of the corresponding sensor nodes in the topological information table.
Step 7. Based on the updated topological information, the optimal paths from the sensor nodes whose data are uncollected to the base station (or gateway) are recalculated, and the optimal path set is updated.

Step 8. Repeat Steps 4 to 7 until the perceived data and residual energy information of all sensor nodes are collected.

In the data collection process, when a sensor node dies, the algorithm returns to Step 2 and reconstructs the network topology. The process flow of data collection is shown in Figure 5. The symbols used in the NEER method and their descriptions are given in Table 5. 


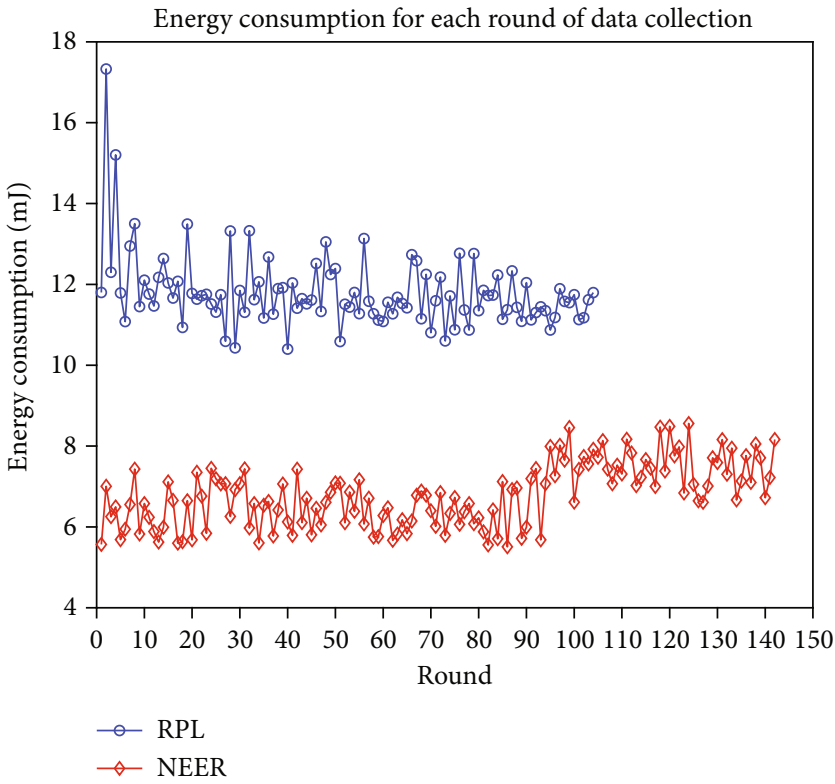

(a)

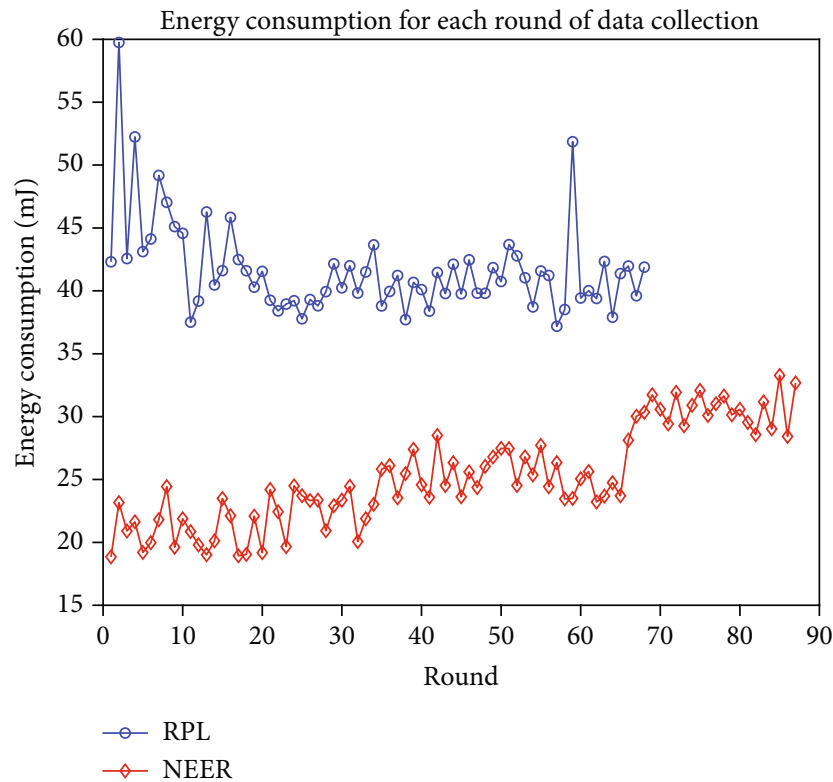

(c)

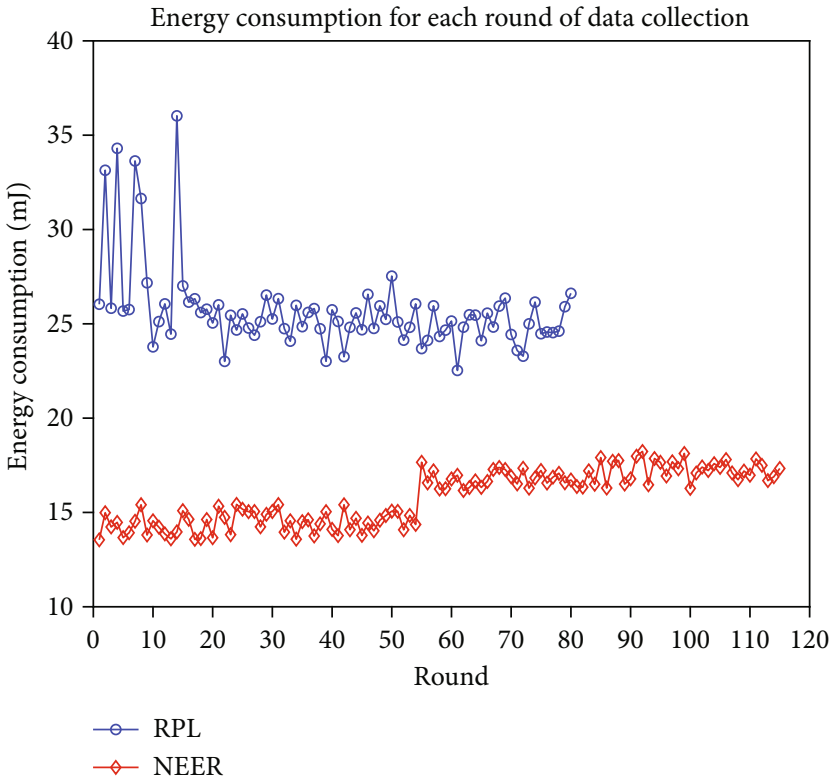

(b)

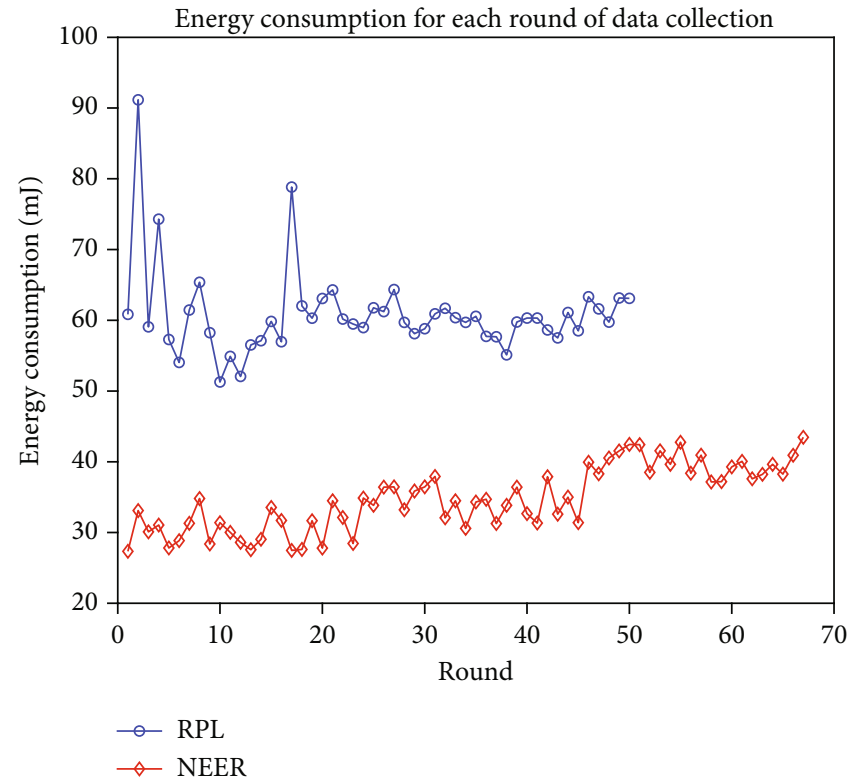

(d)

Figure 9: Comparison of energy consumption of data collection in a single-edge area network: (a) 20 sensor nodes; (b) 40 sensor nodes; (c) 60 sensor nodes; (d) 80 sensor nodes.

4.1.2. Energy Consumption Calculation. From the data collection process, it can be seen that the base station (or gateway) transmits an optimal path to each sensor node on the path, and then, a node starts transmitting data to the base station (or gateway). Let $P=\left\{v_{1} \longrightarrow v_{2}\right.$ $\longrightarrow v_{3} \longrightarrow \cdots \longrightarrow v_{m} \longrightarrow$ base $\}$ be a path from sensor node $v_{1}$ to the base station (or gateway) base; energy consumptions of data transmission are set as weights of edges connecting the path. Except for node $v_{1}$, which receives and sends data only once, the other nodes, $v_{2}, v_{3}, \cdots, v_{m}$, receive and send data twice.
According to the previously described data collection process, the base station (or gateway) transmits the optimal path to each sensor node on the working path, and then, the node starts transmitting data to the base station (or gateway). Let $P=\left\{v_{1} \longrightarrow v_{2} \longrightarrow v_{3} \longrightarrow \cdots \longrightarrow v_{m} \longrightarrow\right.$ base $\}$ be a path from a sensor node $v_{1}$ to the base station (or gateway), and the energy consumptions of the nodes for data transmission are set as the weights of the edges connecting the path. Except for node $v_{1}$, which needs to receive and send data only once, the other nodes, $v_{2}, v_{3}, \cdots, v_{m}$, need to receive and send data twice. 


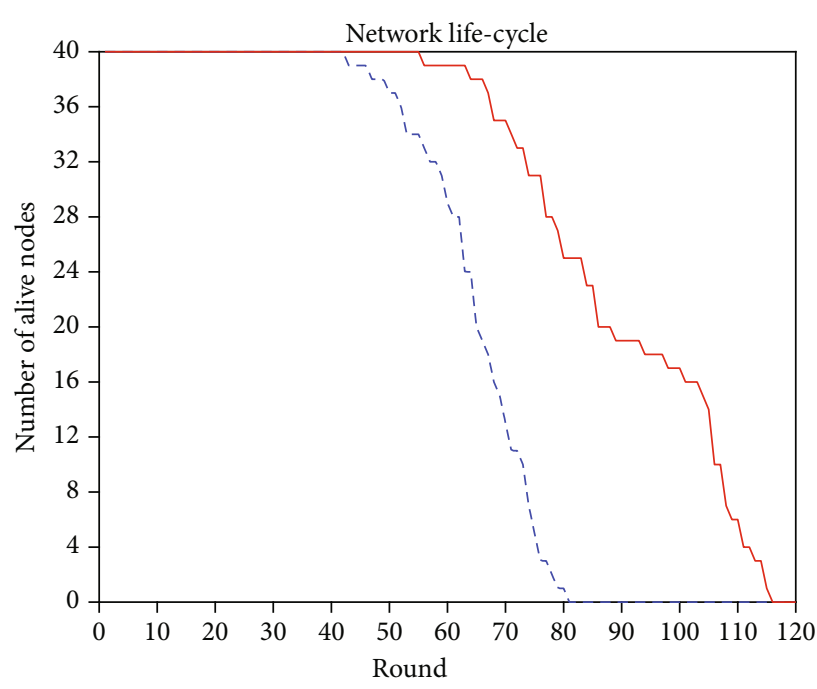

-.- RPL

- NEER

(a)

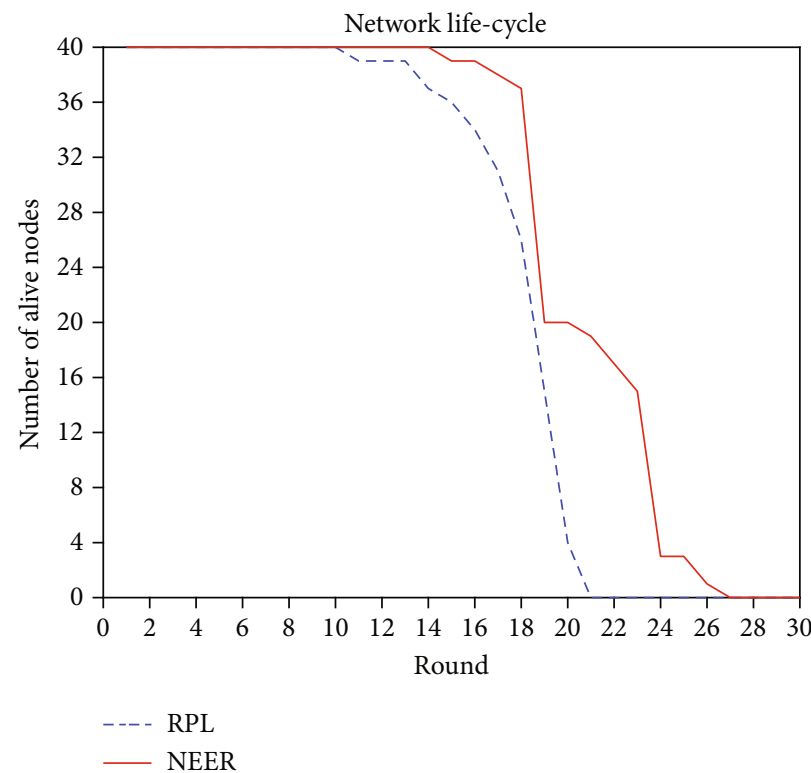

(c)

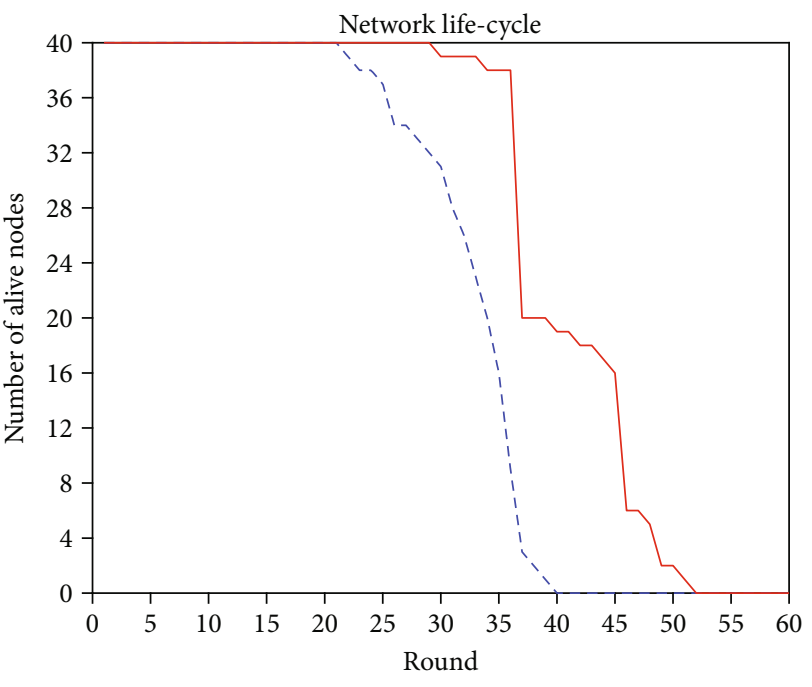

RPL

- NEER

(b)

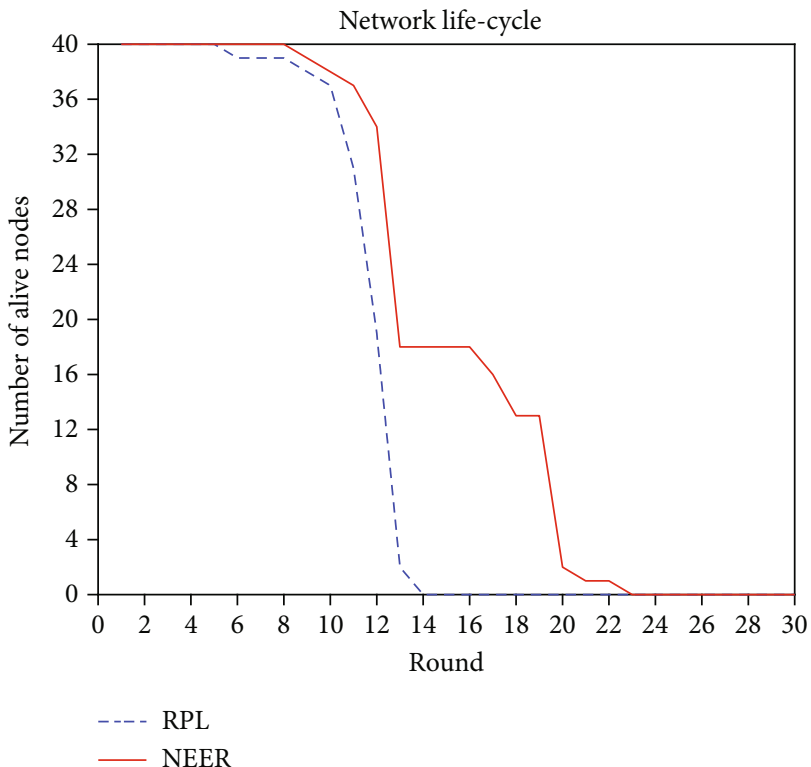

(d)

FIGURE 10: The network life cycle in a single-edge area network: (a) $100 \mathrm{~m} \times 100 \mathrm{~m}$; (b) $200 \mathrm{~m} \times 200 \mathrm{~m}$; (c) $400 \mathrm{~m} \times 400 \mathrm{~m}$; (d) $800 \mathrm{~m} \times 800 \mathrm{~m}$.

Given this setup, the edge weights of the network topological graph can be calculated by

$$
\left\{\begin{array}{l}
E_{\text {start }}=E_{\mathrm{Rx}}(h)+E_{\mathrm{Tx}}(h, d), \\
E_{\text {others }}=2\left[E_{\mathrm{Rx}}(h)+E_{\mathrm{Tx}}(h, d)\right],
\end{array}\right.
$$

where $E_{\text {start }}$ denotes the energy consumption of the initial sensor node, $E_{\text {others }}$ represents the energy consumption of the sensor nodes other than the initial sensor node; $E_{\mathrm{Rx}}(h)$ is the energy consumption associated with data receiving, and $E_{\mathrm{Tx}}(h, d)$ is the energy consumption associated with data transmission, and they can be calculated by Equations (1) and (2), respectively.
4.1.3. Optimal Path Calculation of Edge Area Network. In order to minimize the energy consumption of each data transmission, the path with the least energy consumption is selected as an optimal path for data transmission. The pseudocode of the calculation algorithm of the optimal path from the base station (or gateway) to all sensor nodes is given in Algorithm 2.

It can be seen from the pseudocode of Algorithm 2 that the lines 3-7 need to be executed $n$ times, and the lines 10-11 need to be executed $m+n$ times. The while loop needs to be executed $n$ times. In the worst case, the complexity of the topological model construction algorithm is $O\left(n^{2}+m n\right)$, where $n$ denotes the number of sensor nodes and $m$ denotes the number of edges. 


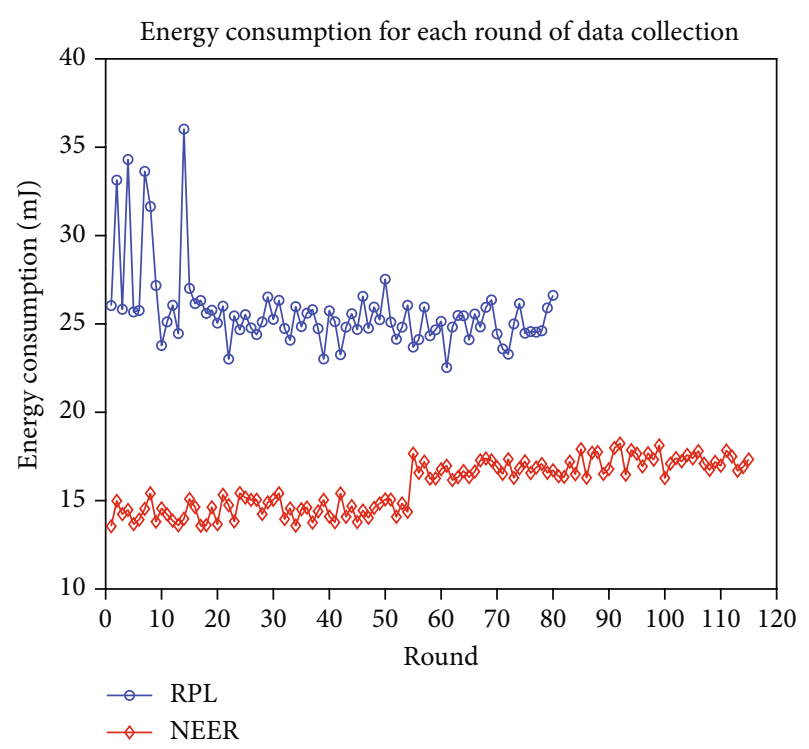

(a)

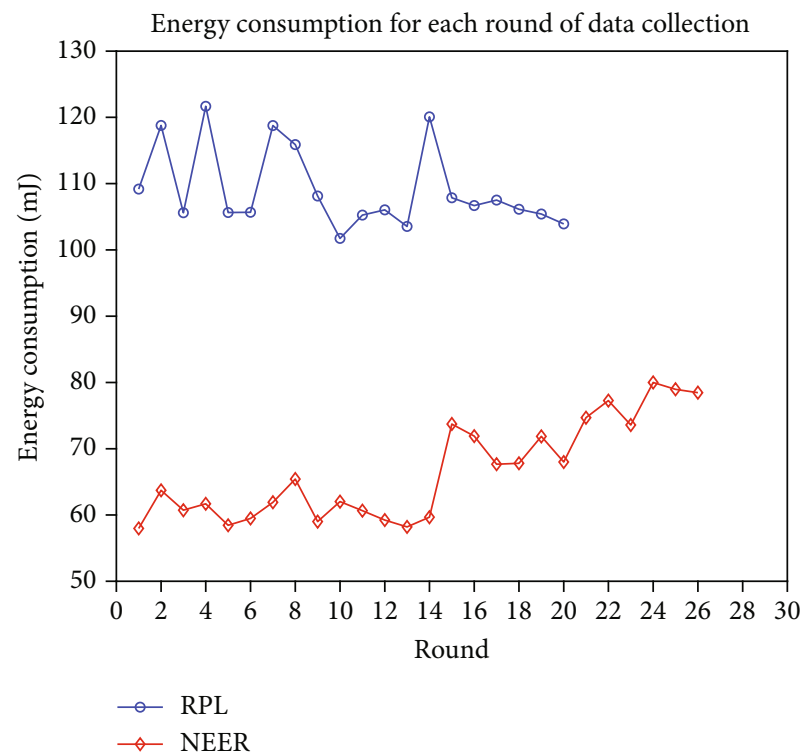

(c)

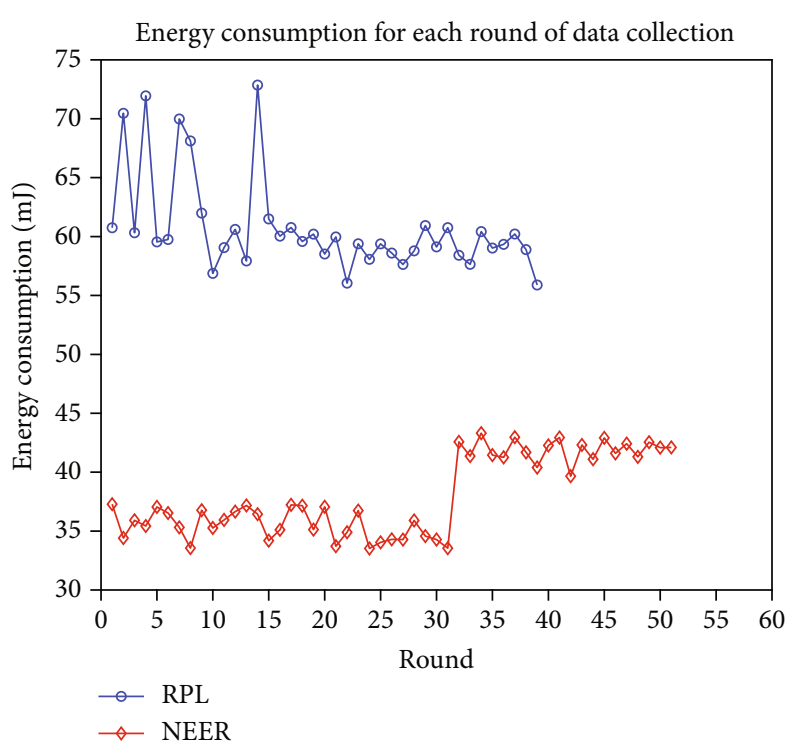

(b)

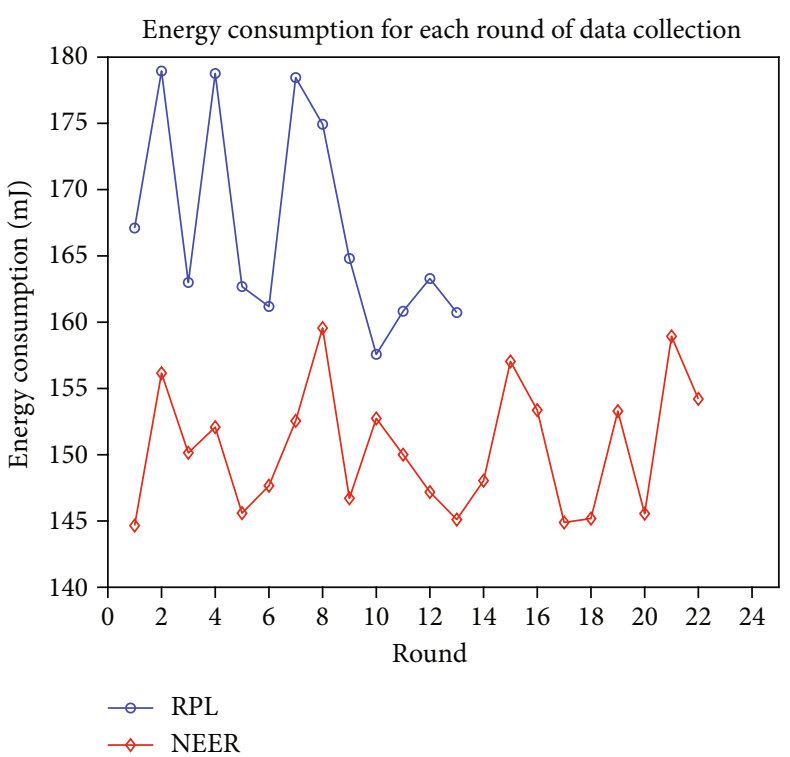

(d)

Figure 11: Comparison of energy consumption of data collection in a single-edge area network: (a) $100 \mathrm{~m} \times 100 \mathrm{~m}$; (b) $200 \mathrm{~m} \times 200 \mathrm{~m}$; (c) $400 \mathrm{~m} \times 400 \mathrm{~m}$; (d) $800 \mathrm{~m} \times 800 \mathrm{~m}$.

The specific steps of Algorithm 2 are as follows:

Step 1. Initialize the calculation process: set the base station (or gateway) number as $v_{0}$ and sensor node number as $v_{i}$. Initially, let $S=\left\{v_{0}\right\}$ and $T=\{V-S\}$. The initial energy of each sensor node is set to $e_{i}$. The value of the side weight formed by the sensor node in $T$ satisfies the following conditions: if $v_{0}$ is adjacent to $v_{i}, c_{0 i}$ is the energy consumption value of data transmitted from the base station (or gateway) $v_{0}$ to the sensor node $v_{i}$, and if the energy consumption value is greater than the residual energy of the node, then $c_{0 i}$ is infinite; otherwise, set $e_{i}=r_{i}$.

If $v_{0}$ and $v_{i}$ are not adjacent to each other, then $c_{0 i}$ is infinite, where $c_{0}=2\left[E_{\mathrm{Rx}}(h)+E_{\mathrm{Tx}}(h, d)\right]$ and $r_{i}=e_{i}-c_{0 i}$.
Step 2. The sensor node $v_{\min }$ with the smallest weight is selected from $T$ and added to $S$; then, the residual energies of all the forwarded sensor nodes are updated.

Step 3. The weight values of the remaining sensor nodes in $T$ are updated: if the value of the side weight from $v_{0}$ to $v_{i}$ decreases after adding $v_{\min }$ as an intermediate node, then modify the value of the side weight.

Step 4. Repeat Steps 2 to 3 until all sensor nodes are included in $S$ (i.e., $V=S$ ), and then, stop the calculation process.

4.2. NEER Analysis. The NEER is a semicentralized routing method. The execution of the NEER algorithm is performed in the base station (or gateway) of each edge area network 


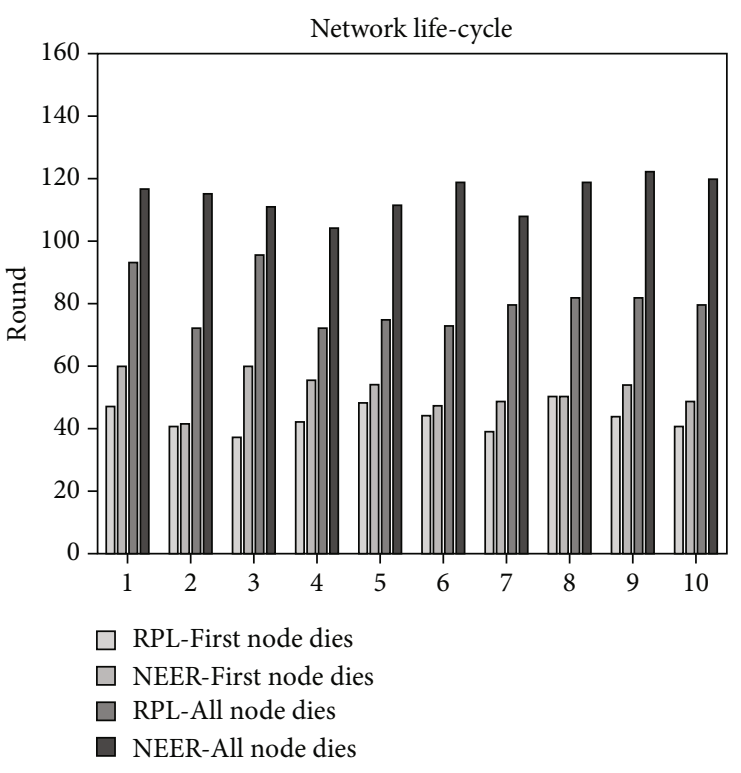

(a)

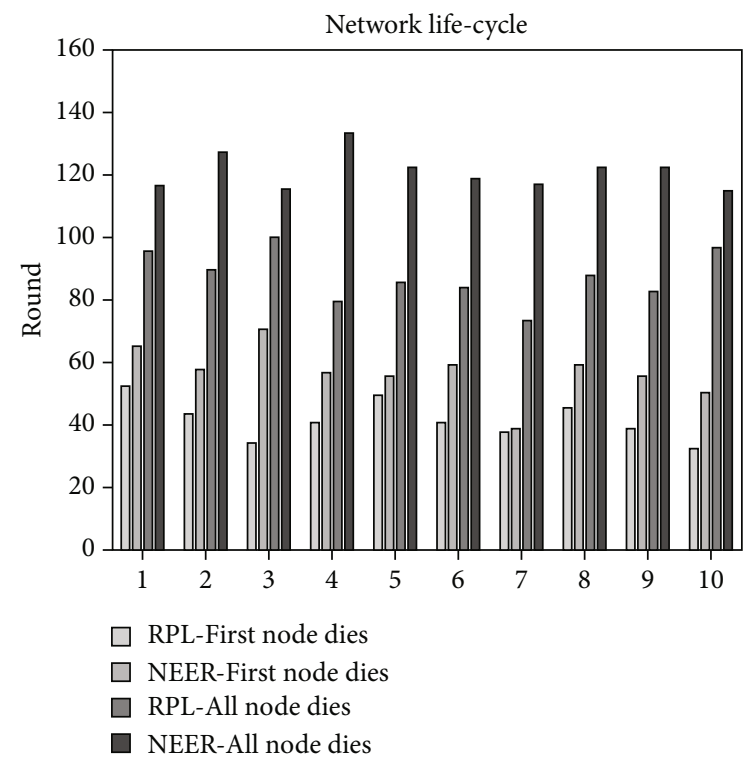

(c)

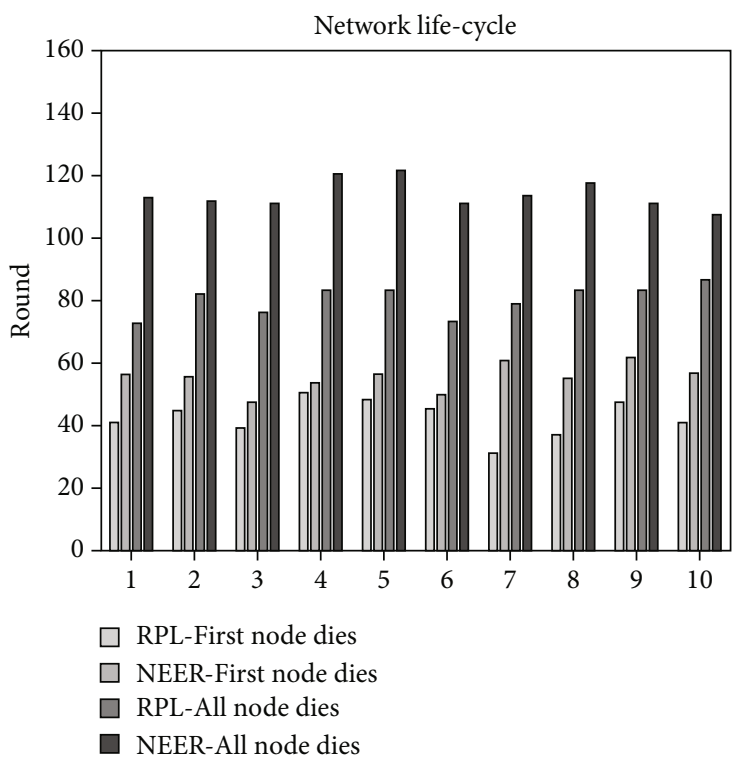

(b)

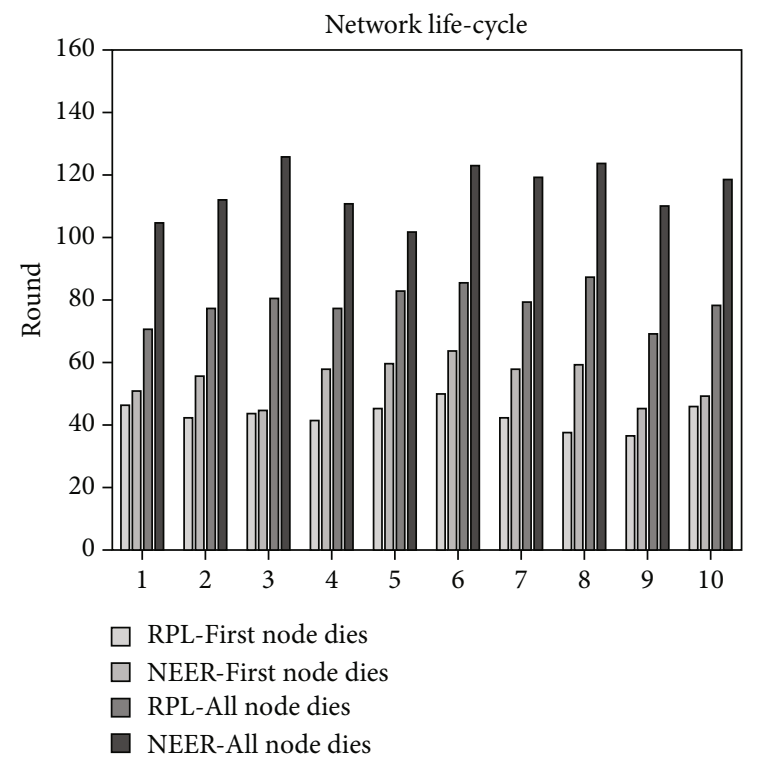

(d)

FIgURe 12: Network life cycle under different initial energy distributions in a single-edge area network: (a) Poisson distribution; (b) binomial distribution; (c) normal distribution; (d) uniform distribution.

with good calculation performance and sufficient energy supply. As mentioned before, sensor nodes can perform only simple operations, such as data storage and data transmission and reception. Thus, the proposed design reduces the energy consumption of sensor nodes. In addition, the residual energy of sensor nodes is considered in the processes of data transmission and routing. Data are transmitted along a route with minimum energy consumption, which ensures that the minimum energy is consumed in each transmission and, thereby, the life-cycle of the network is prolonged. The performance of the proposed method is proven by the following proposition.

Assume that $V$ denotes a set of all sensor nodes and the base station (or gateway) in the edge area network, and $S$ is the collection of nodes that have found their optimal paths, i.e., paths with minimum energy consumption. In the initial state, in $S$, there is only the base station (or gateway) $v_{0} \in S$, and $T$ is the set of nodes for which the minimum energy paths have not been found yet; thus, $T=V-S$.

Proposition 1. Assume that $v_{1}, v_{2}, \cdots, v_{m}$ correspond to $m$ sensor nodes from $T$ for which optimal paths have been successively found using Algorithm 2, and $P_{v_{1}}, P_{v_{2}}, \cdots, P_{v_{m}}$ denote the minimum energy consumptions of data transmissions from the base station (or gateway), $v_{0}$, to different nodes. Then, find another node $v_{m+1}$ from $T$, whereas $P_{v_{m+1}}$ determined by Algorithm 2 corresponds to the minimum 


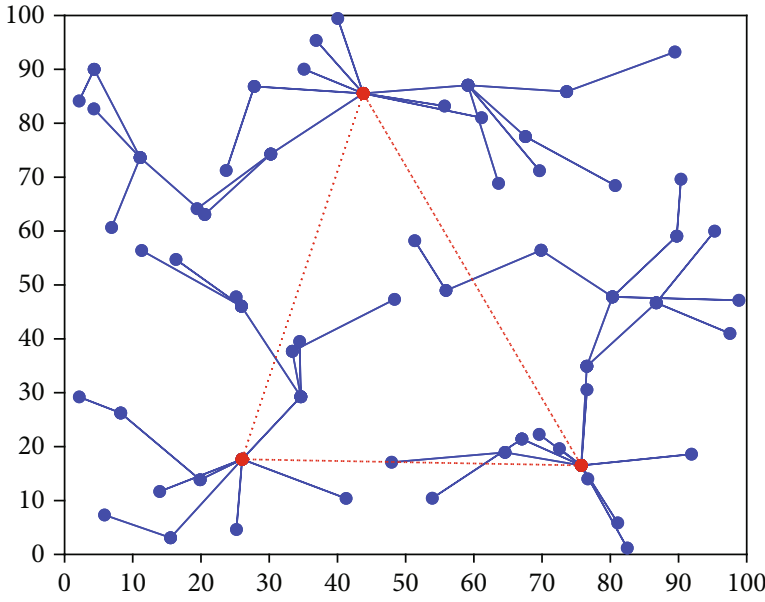

(a)

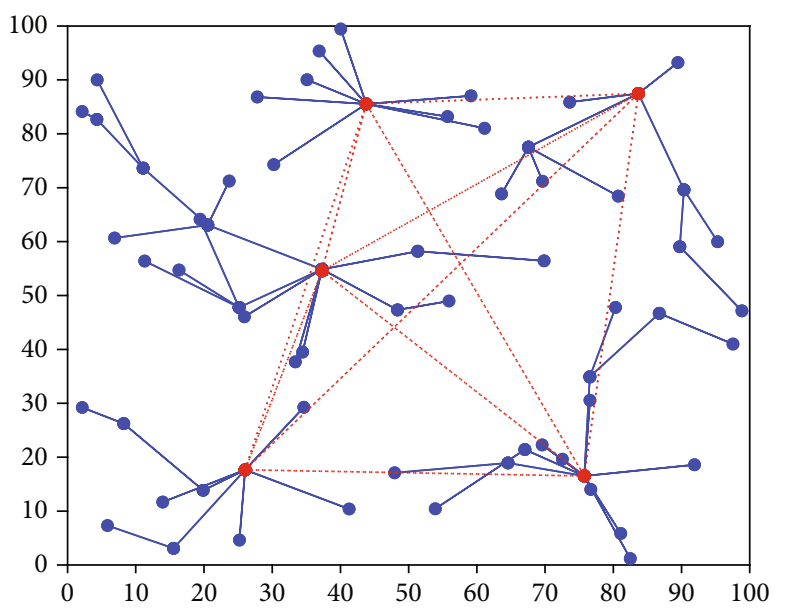

(c)

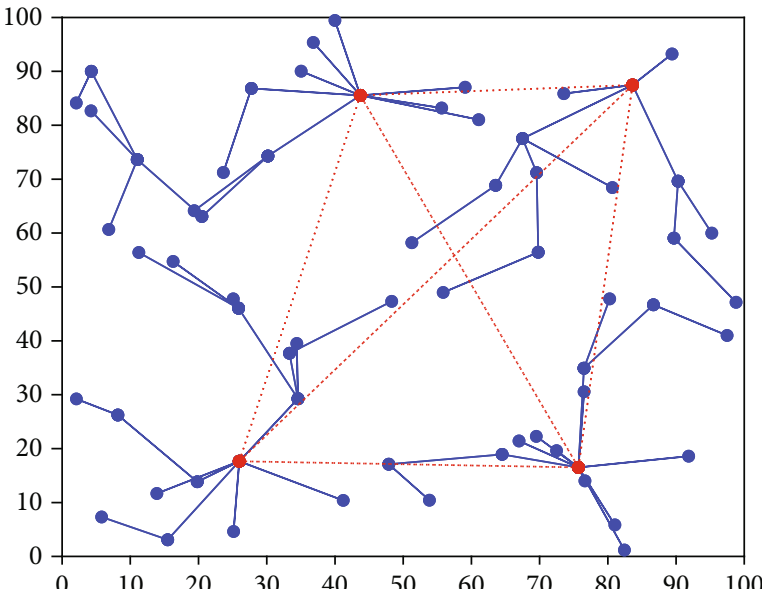

(b)

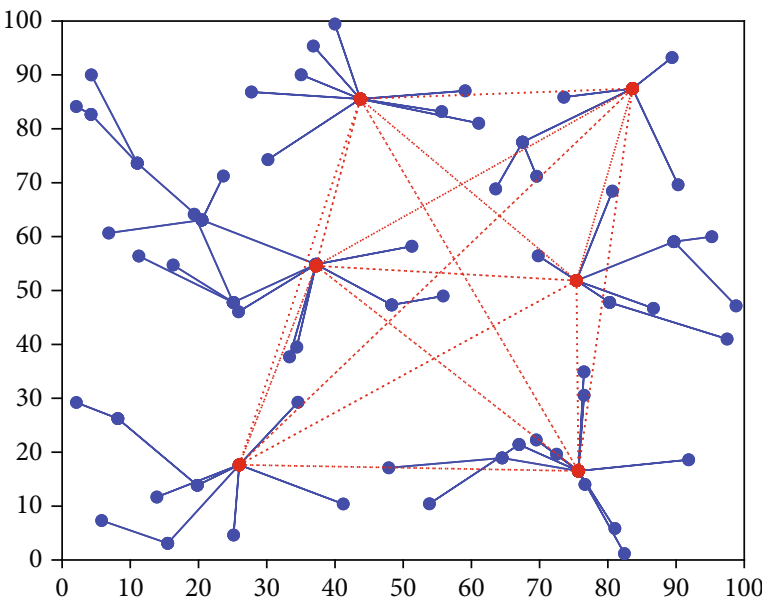

(d)

FIGURE 13: Topological structures of multiple-edge area networks constructed by the RPL: (a) three base station nodes; (b) four base station nodes; (c) five base station nodes; (d) six base station nodes.

energy consumption from the base station (or gateway) to node $v_{m+1}$.

Proof. Assume that $P_{v_{m+1}}$ is not the minimum energy consumption from the base station (or gateway) to a node $v_{m+1}$, and let $U$ be the set of nodes through which the minimum energy consumption path passes from the base station (or gateway) $v_{0}$ to the node $v_{m+1}$. Let the energy consumption along this path be $P$ such that $P<P_{v_{m+1}}$.

As $U \cap T \neq \varnothing, o \in U$, and $o \in S \Rightarrow U \cup S \neq \varnothing$, the node that is closest to $v_{m+1}$ and does not belong to $S$ but is on the path with minimum energy consumption from the base station (or gateway) $v_{0}$ to the node $v_{m+1}$ is set as $v_{x}$; the immediate successor of $v_{x}$ is $v_{y}$.

Further, as the weights in a WSN indicate the consumption of the energy and they are all positive, then it holds that $P_{v_{x}}<P_{v_{y}}<P_{v_{m+1}}=P$. The equality sign is established when $v_{y}$ is $v_{m+1}$. Since $v_{x} \notin S \Longrightarrow P_{v_{x}}>P_{v_{m+1}}$, this conflicts with the formal inequality. Thus, the hypothesis is not established, and the proposition is proved by contradiction.

\section{Simulation Experiments}

In order to verify the effectiveness of the proposed method, the Contiki/Cooja $[38,39]$ platform was selected for simulation experiments, and the proposed method was compared with the existing RPL protocol. The RPL protocol is used in intelligent object networks. It was designed by Internet Engineering Task Force (IETF) for the interconnection of restricted devices through lossy links. Contiki is an opensource and event-driven operating platform that enables the interoperability of low-power, energy-constrained, and memory-constrained microdevices.

The proposed NEER method was compared with the existing RPL regarding two indexes, the entire network lifecycle and energy consumption. The network life cycle was measured by the number of rounds of data acquisition when the first sensor node died and when all sensor nodes died. It 


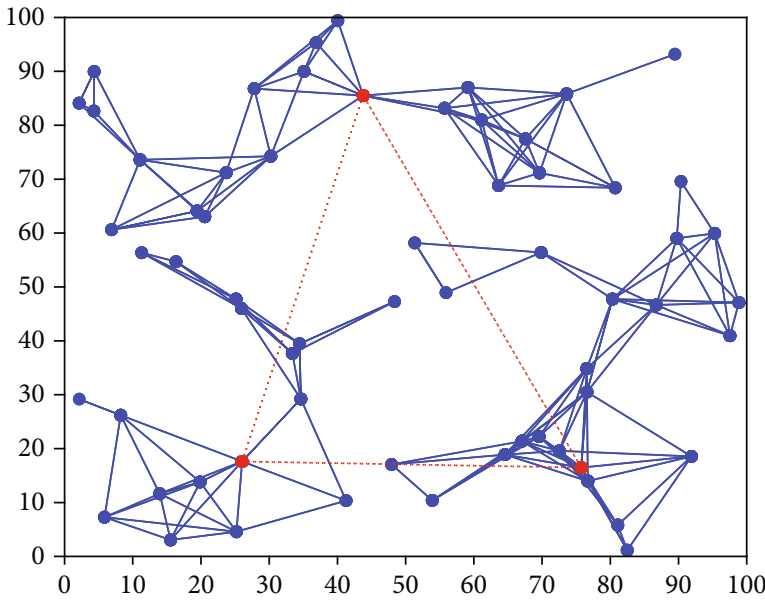

(a)

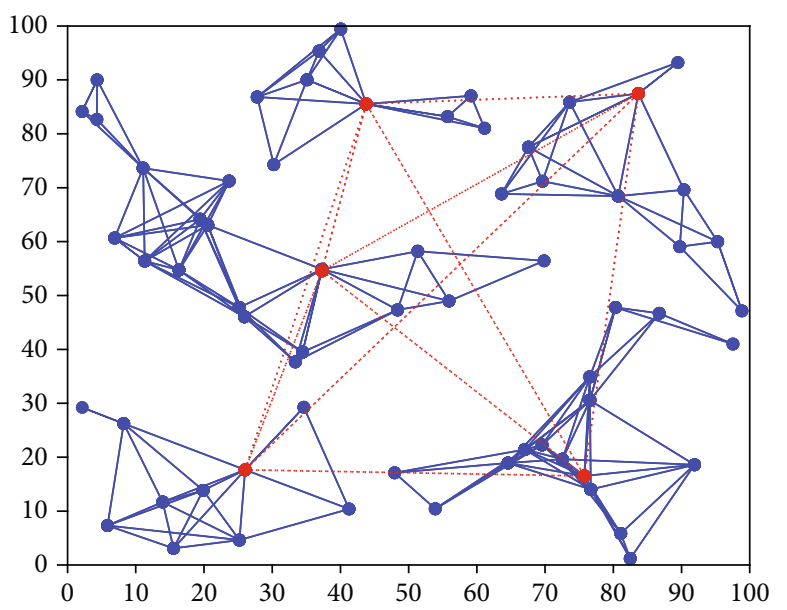

(c)

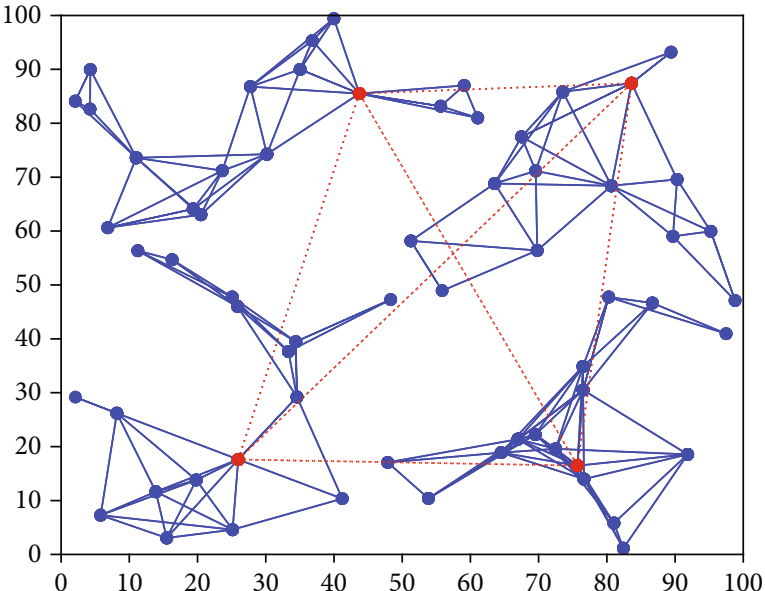

(b)

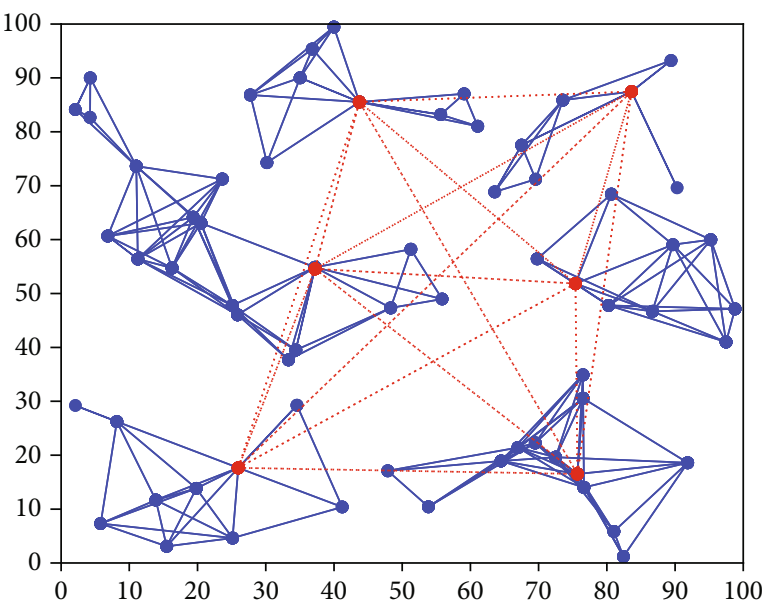

(d)

FIGURE 14: Topological structures of multiple-edge area networks constructed by the NEER: (a) three base station nodes; (b) four base station nodes; (c) five base station nodes; (d) six base station nodes.

was considered that a sensor node died when the residual energy of the sensor node was less than or equal to zero.

5.1. Experimental Setup. Two groups of simulation experiments were conducted. The first group of experiments included the experiments with a single-edge area network, which included only one base station node. The effectiveness of the NEER method was verified and compared to that of the RPL from three aspects: the number of nodes, network area size, and initial energy distribution. The second group of experiments included the experiments with multipleedge area networks, which included multiple base station nodes. The influence of the number and location of different base station nodes on the NEER performance was studied and compared with that of the RPL.

In these two groups of experiments, the basic simulation parameters were set as follows:

(1) The energy consumption sent and received by a sensor node was $E_{\mathrm{Tx}-\mathrm{elec}}=E_{\mathrm{Rx}-\mathrm{elec}}=E_{\text {elec }}=50 \mathrm{~nJ} / \mathrm{bit}$
(2) The energy consumption of the sensor node amplification was $\varepsilon_{\mathrm{amp}}=100 \mathrm{pJ} / \mathrm{bit} / \mathrm{m}^{2}$

(3) The number of bits per transmission packet was 1000 bits

The experiments were carried out in two stages. In the first stage, the topological model was constructed using Algorithm 1, and in the second stage, the perceived data were routed using the proposed NEER method. Additionally, the second stage was divided into several rounds, whereas the round was considered to be a period within which the data collection of all nodes was conducted.

\subsection{Results and Discussion}

\subsubsection{Single-Edge Area Network Experiment}

(1) Effect of Sensor Node Number. This experiment was performed to analyze the effect of the number of sensor nodes on the NEER method's performance and compared it with that of the RPL. In the area with a size of $100 \mathrm{~m} \times 100 \mathrm{~m}$, 


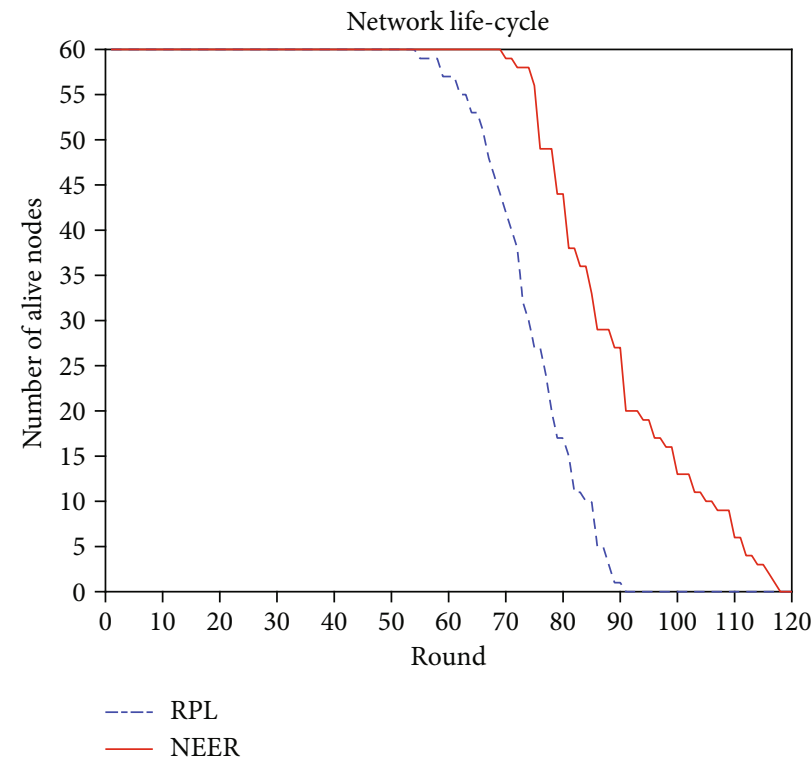

(a)

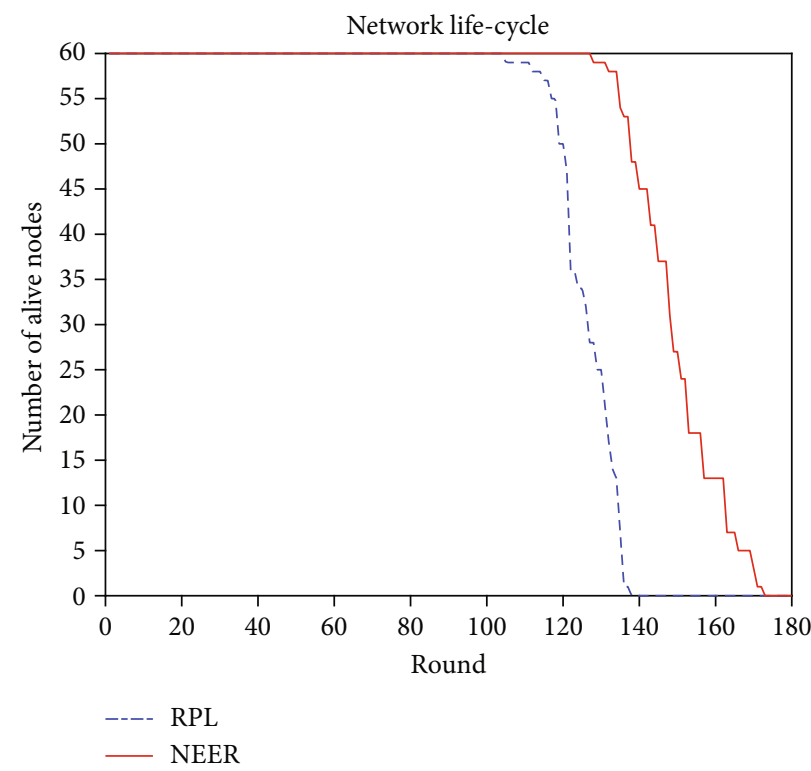

(c)

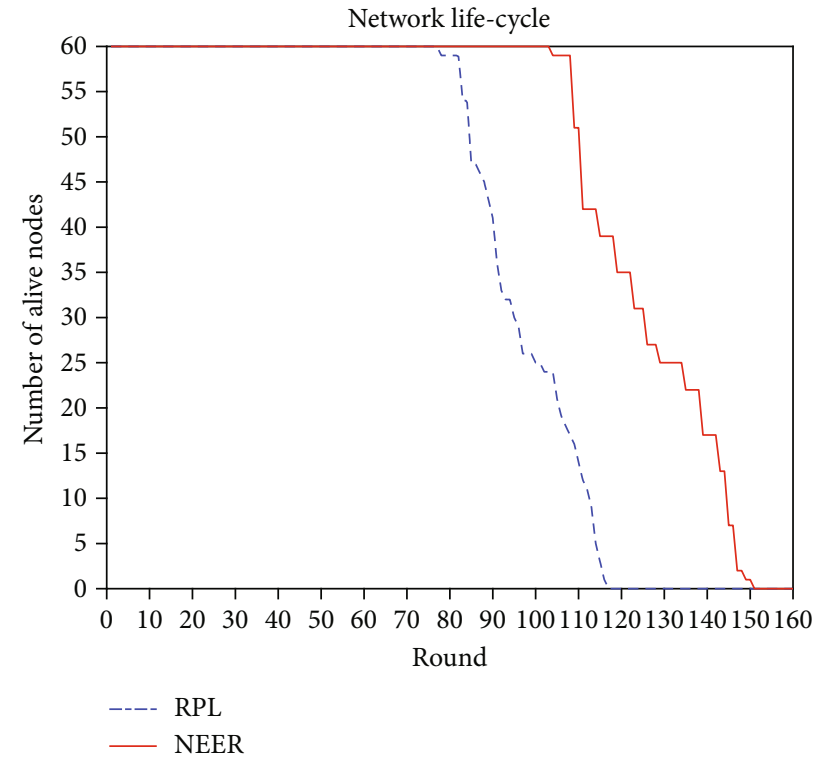

(b)

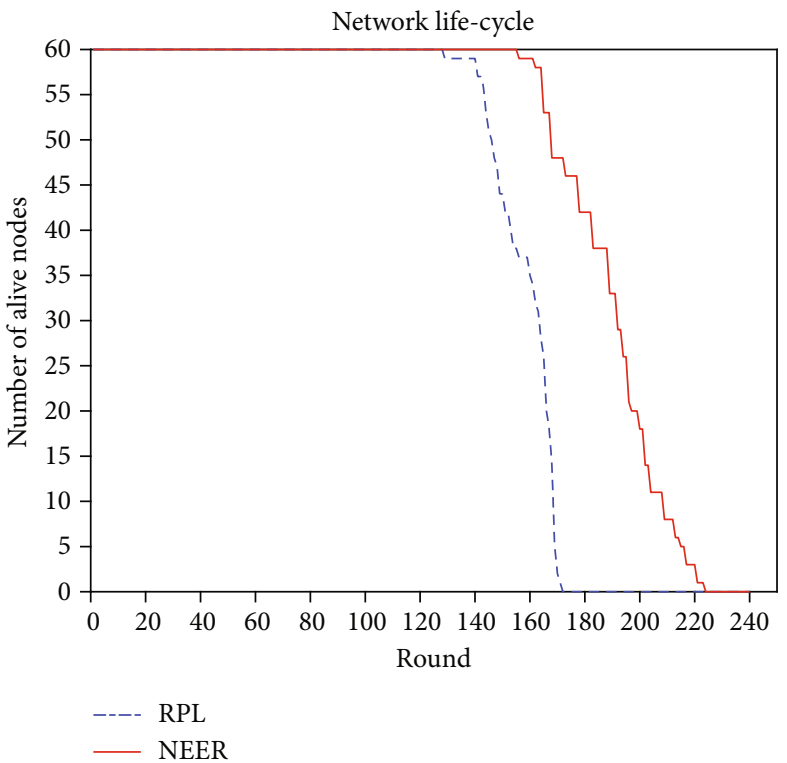

(d)

Figure 15: The network life cycle in multiple-edge area networks: (a) three base station nodes; (b) four base station nodes; (c) five base station nodes; (d) six base station nodes.

one base station node and different numbers of sensor nodes were randomly deployed; the number of sensor nodes was set to $20,40,60$, and 80 . These numbers were chosen based on the previous analysis of node number regarding node number and deployment influence on the network life and energy consumption. In the experiment, the only parameter which was changed was the number of sensor nodes. The upper limit of the transmission range of the sensor nodes was set to $20 \mathrm{~m}$, and the initial energy of the sensor nodes was $100 \mathrm{~mJ}$.

The network topologies consisting of 20,30,40, and 80 sensor nodes generated by the RPL protocol are presented in Figures 6(a)-6(d), and those generated by the proposed
NEER method are shown in Figures $7(\mathrm{a})-7(\mathrm{~d})$. In Figures 6 and 7, red dots represent the base stations (or gateways).

The network life cycle for different numbers of sensor nodes of the two methods is presented in Figures 8(a)8(d). As shown in Figure 8, at the number of sensor nodes of $20,40,60$, and 80 , the first sensor node of the RPL protocol died when the number of rounds of data collection in the network reached the value of $64,43,28$, and 19 , respectively, while the first sensor node of the NEER died after 95, 56, 37, and 25 rounds of data collection, respectively. In addition, at the number of sensor nodes of $20,40,60$, and 80 , all sensor nodes of the RPL protocol died when the number of rounds 


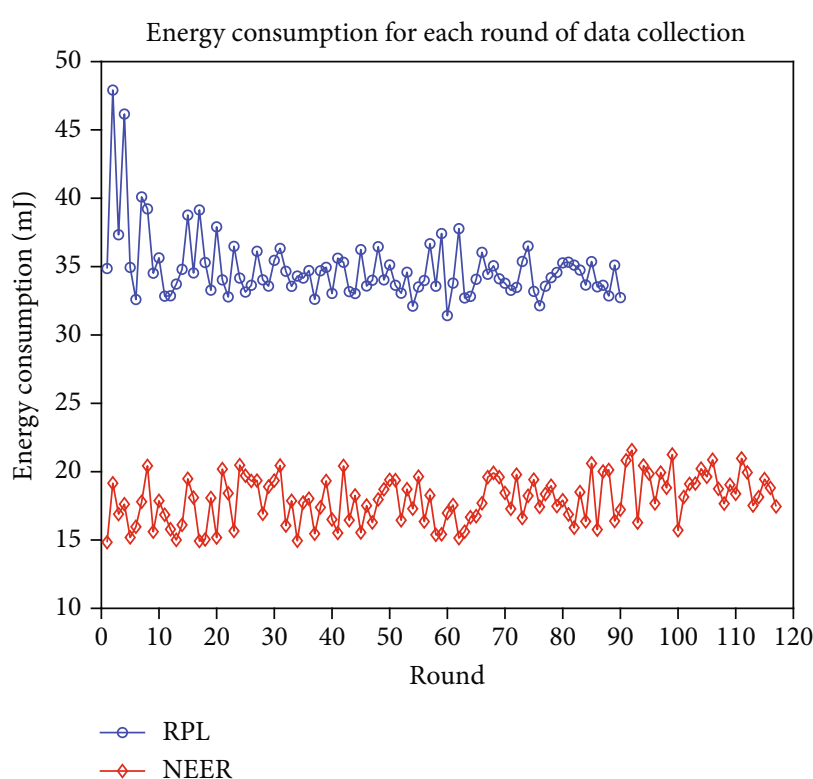

(a)

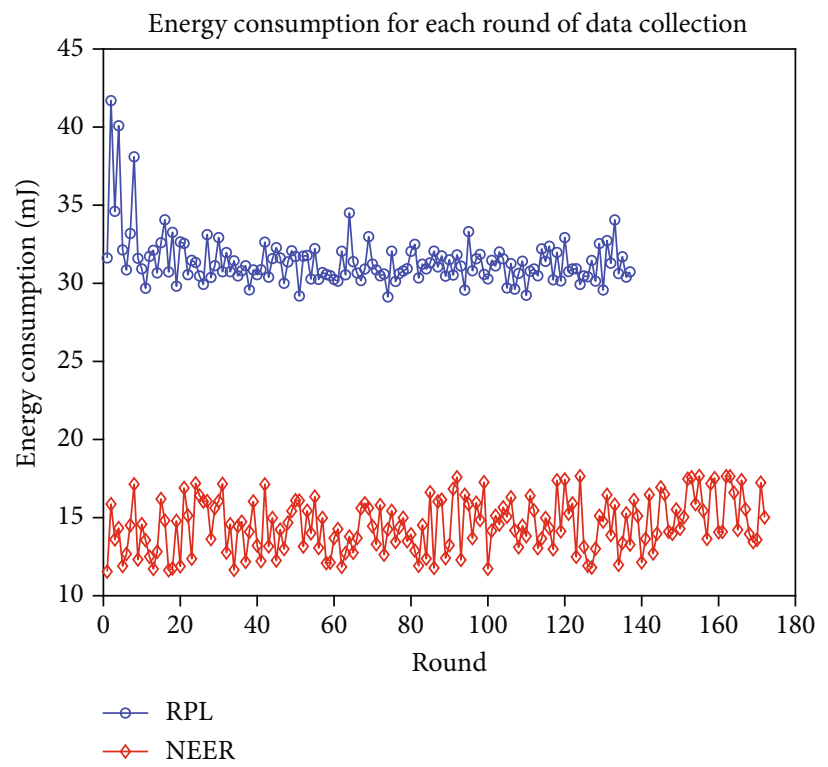

(c)

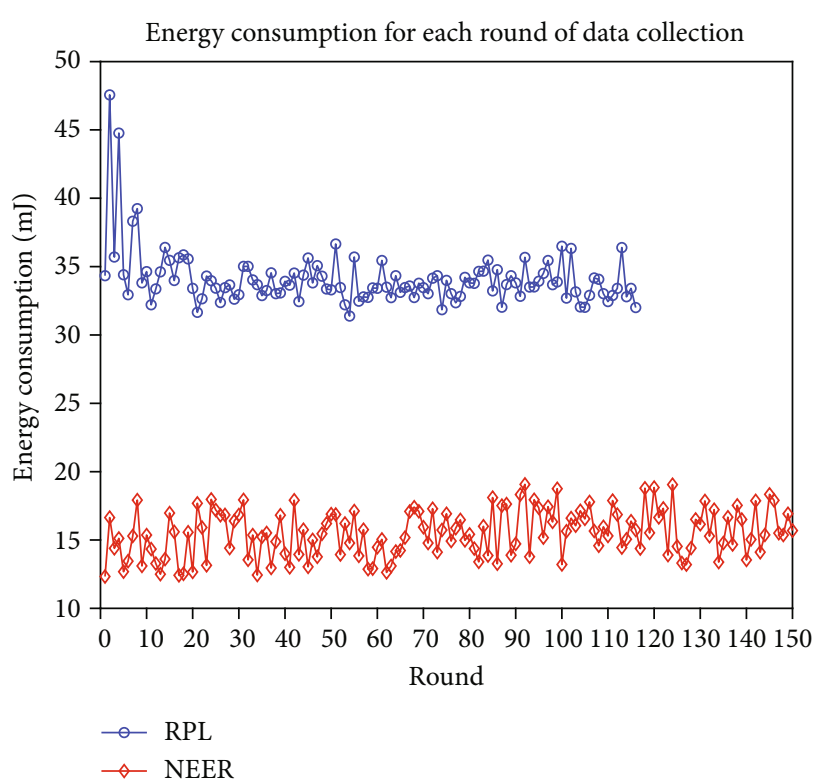

(b)

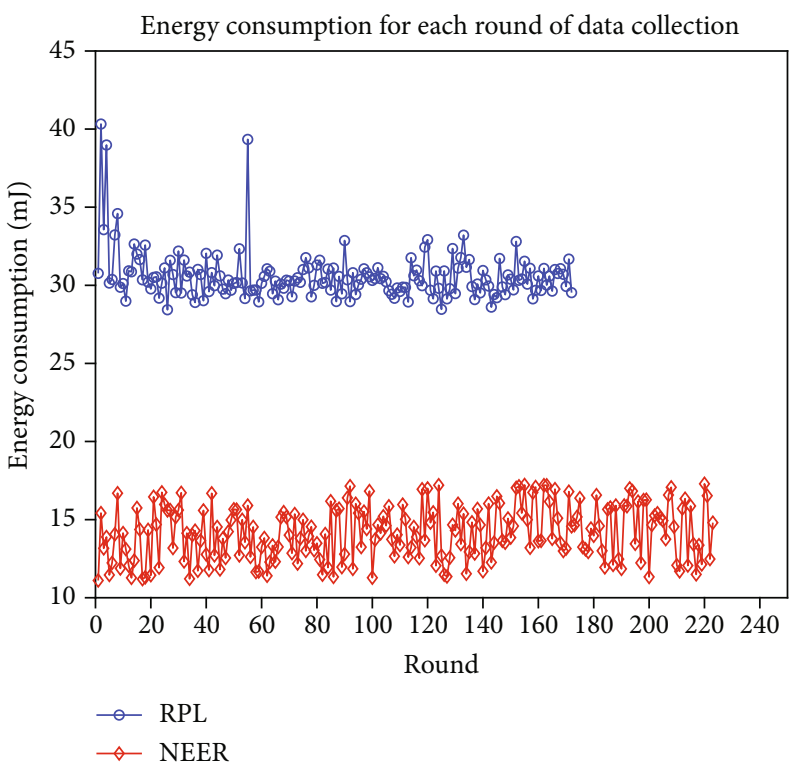

(d)

FIgURE 16: Comparison of energy consumption of data collection in multiple-edge area networks: (a) three base station nodes; (b) four base station nodes; (c) five base station nodes; (d) six base station nodes.

of data collection in the network reached the values of 105 , 81,69 , and 51, respectively, while all sensor nodes of the NEER method died after 143, 116, 88, and 68 rounds of data collection, respectively. Therefore, the NEER method prolonged the life cycle of the network. This was mainly because there were multiple paths between sensor nodes in the network topology constructed by the NEER method compared with the RPL, and the optimal path was selected dynamically during the data transmission process according to the residual energy of nodes.

The energy consumption in each round of data acquisition of a single-edge area network composed of $20,30,40$, and 80 sensor nodes of the two methods is presented in
Figures 9(a)-9(d), respectively. As shown in Figure 9, the NEER method consumed less energy during data collection than the RPL protocol. This was because, in the NEER method, sensor nodes did not need to implement a routing algorithm and did not save routing information, which was all completed at the base station. The sensor nodes performed only data transmission according to the routing path defined by the base station; meanwhile, the data sensed by all sensor nodes on the path were collected. This not only saved computing and storage resources but also reduced energy consumption. In addition, the RPL protocol line was shorter than that of the NEER method, as shown in Figure 9. This was because all sensor nodes in the RPL died earlier than 


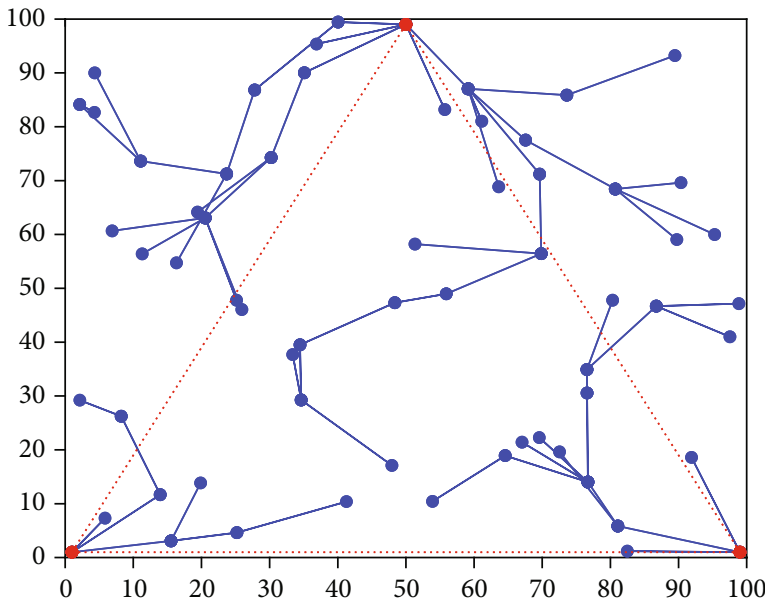

(a)

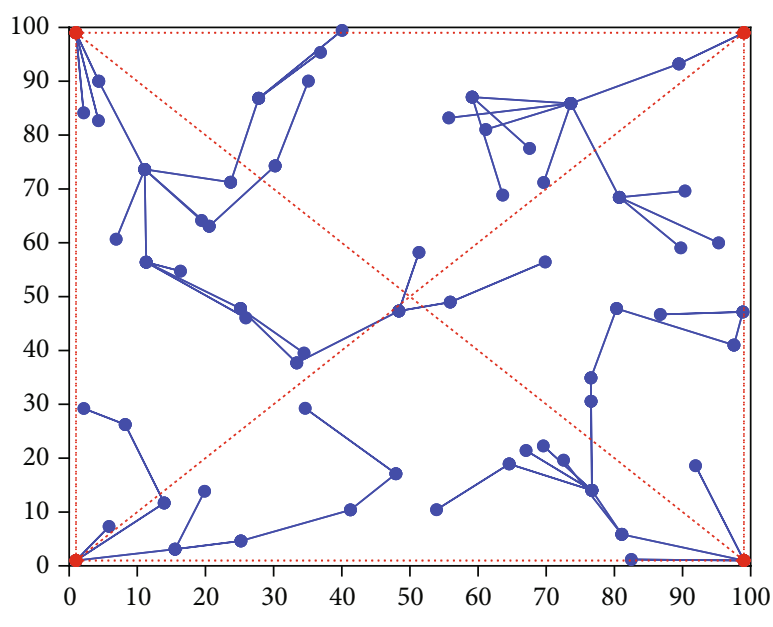

(c)

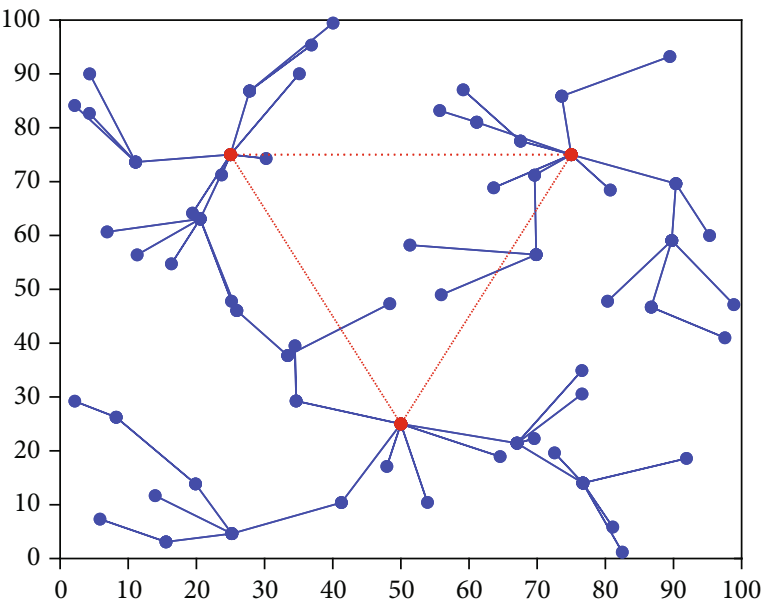

(b)

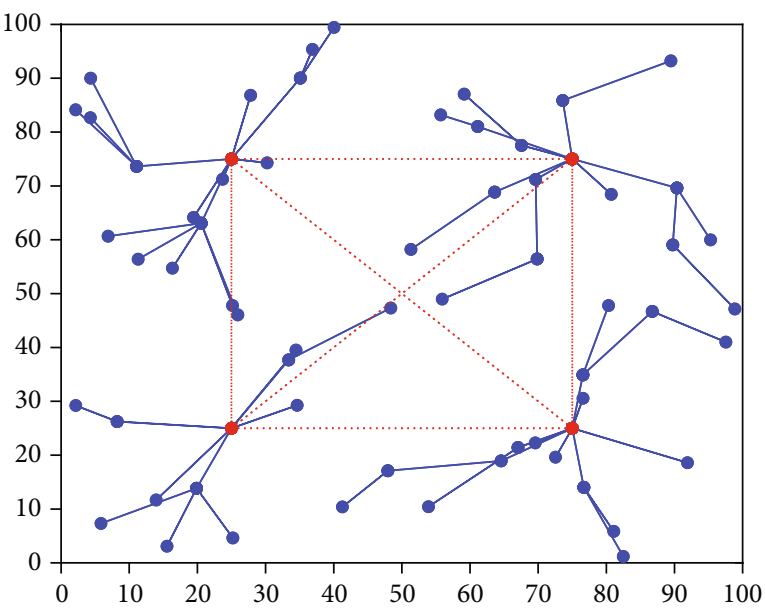

(d)

FIgURE 17: Topological structures of multiple-edge area networks constructed by the RPL: (a) position 1 with three base stations; (b) position 2 with three base stations; (c) position 1 with four base stations; (d) position 2 with four base stations.

those in the NEER, so there was no energy consumption data after that. This further validated that the NEER method could prolong the life cycle of the network.

(2) Effect of Network Area Size. This experiment was conducted to analyze the effect of the network area size on the NEER method performance, as well as on that of the RPL protocol. One base station node and 40 sensor nodes were randomly deployed in an area of a different size. The network area size was set to $100 \mathrm{~m} \times 100 \mathrm{~m}, 200 \mathrm{~m} \times 200 \mathrm{~m}$, $400 \mathrm{~m} \times 400 \mathrm{~m}$, and $800 \mathrm{~m} \times 800 \mathrm{~m}$. The network sizes were chosen based on the same criterion as the node numbers. In the experiment, only the network area size was changed, while all other parameters were fixed. The initial energy of the sensor nodes was $100 \mathrm{~mJ}$. The topological structure of the network was the same as that presented in Figures 6(b) and $7(b)$.

The network lifecycle and energy consumption of the two methods for a single-edge area network with a different network area size are presented in Figures 10(a)-10(d) and Figures $11(\mathrm{a})-11(\mathrm{~d})$, respectively. As shown in Figures 10 and 11, for both methods, the network life cycle decreased, and the energy consumption increased with the increase in the network area size.

The reason was that in the case of the same network topological structure, increasing the network area size was equivalent to increasing the communication distance between sensor nodes, so the required transmitting and receiving powers of the nodes would increase; thus, the energy consumption of data transmission would also increase, thereby reducing the life of the network. However, it should be noted that the proposed NEER method had a longer network life and lower energy consumption of data transmission compared with the RPL under the same network area size.

(3) Effect of Initial Energy Heterogeneity. This experiment was conducted to examine the effect of the initial energy heterogeneity on the NEER method and compare it with that on the RPL. One base station node and 40 sensor nodes were randomly deployed in an area of $100 \mathrm{~m} \times 100 \mathrm{~m}$. In this experiment, only the initial energy distribution of sensor 


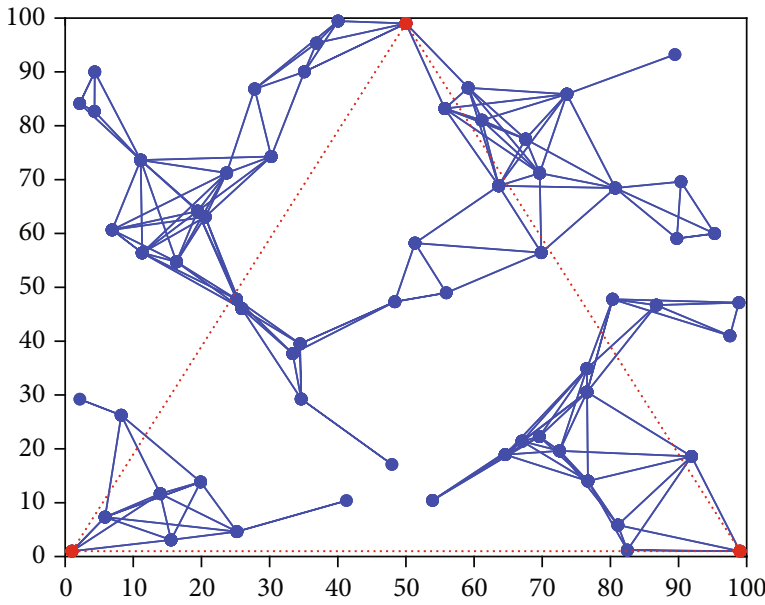

(a)

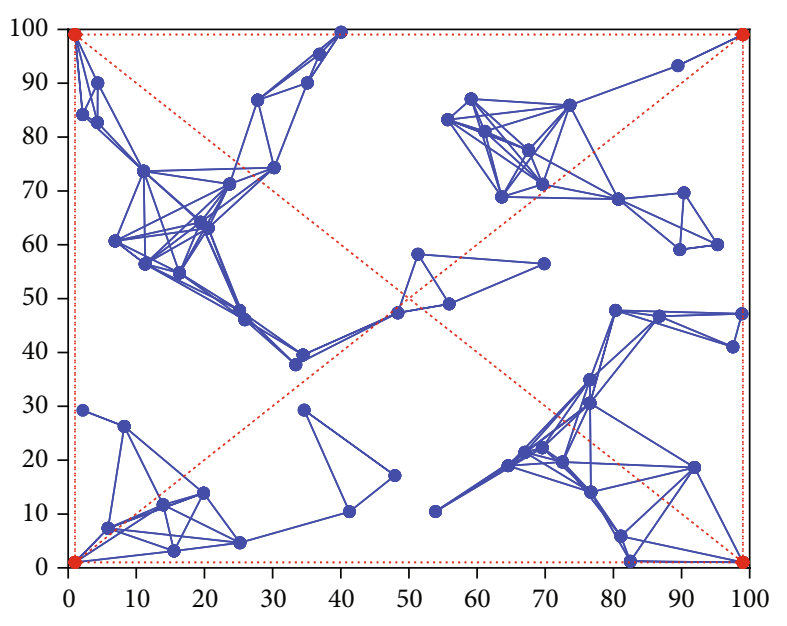

(c)

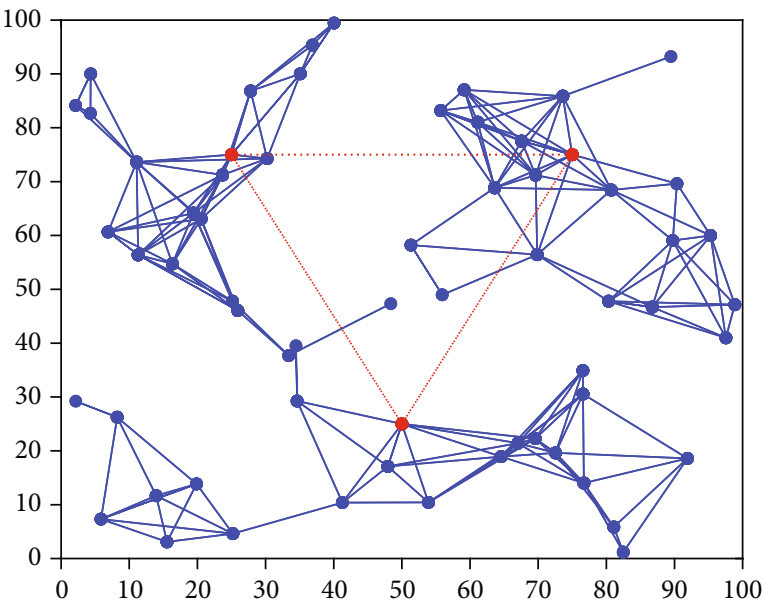

(b)

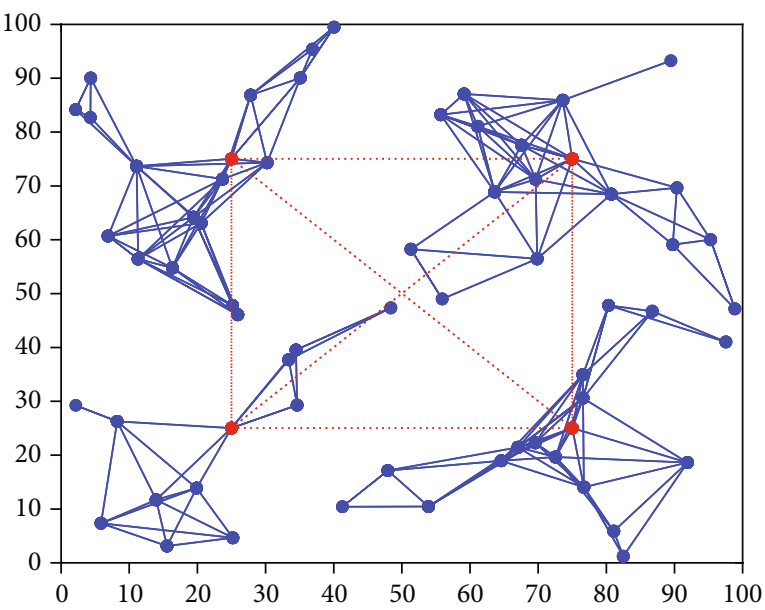

(d)

FIgURE 18: Topological structures of multiple-edge area networks constructed by the NEER: (a) position 1 with three base stations; (b) position 2 with three base stations; (c) position 1 with four base stations; (d) position 2 with four base stations.

nodes was changed, while the other parameters were fixed. The upper limit of the transmission range of the sensor nodes was set to $20 \mathrm{~m}$, and the initial energy of the sensor nodes was different; namely, the initial energy distribution was set to the Poisson distribution, binomial distribution, normal distribution, and uniform distribution, successively. The network topology was the same as that in Figures 6(b) and $7(b)$. For each distribution of the initial energy, ten experiments were conducted, and the results of each of them are shown in Figure 12. In Figure 12, the round numbers corresponding to the death of the first node and the death of all the nodes are presented. As shown in Figure 12, regardless of the initial energy distribution, the lifetime of the network using the NEER was longer than using the RPL protocol.

\subsubsection{Multiple-Edge Area Network Experiment}

(1) Effect of Base Station Number. This experiment was conducted to examine the effect of the number of base stations on the NEER method performance. Also, in all other exper- iments, the performance of the proposed method was compared with that of the RPL protocol. Three, four, five, and six base station nodes and 60 sensor nodes were randomly deployed in an area of $100 \mathrm{~m} \times 100 \mathrm{~m}$. In this experiment, only the number of base station nodes was changed, while the other parameters were kept fixed. The upper limit of the transmission range of the sensor nodes was set to $20 \mathrm{~m}$, and the initial energy of the sensor nodes was $100 \mathrm{~mJ}$. The topologies of multiple-edge area networks generated by the RPL protocol and the proposed method are presented in Figures 13(a)-13(d) and Figures 14(a)-14(d), respectively. In Figures 13 and 14, red dots represent the base stations (or gateways).

The comparison results of network lifetime and energy consumption of the RPL and NEER routing methods for multiple-edge area networks having three, four, five, and six base stations are presented in Figures 15(a)-15(d) and Figures 16(a)-16(d), respectively. As shown in Figures 15 and 16 , as the number of base stations increased, the life of the network prolonged, and energy consumption reduced. Since the WSN was divided into multiple-edge area 


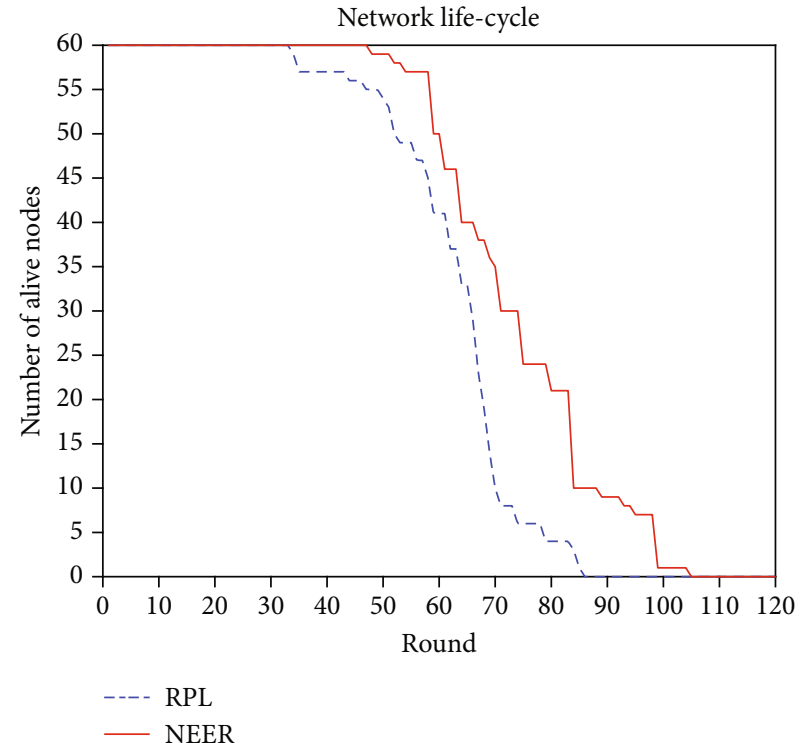

(a)

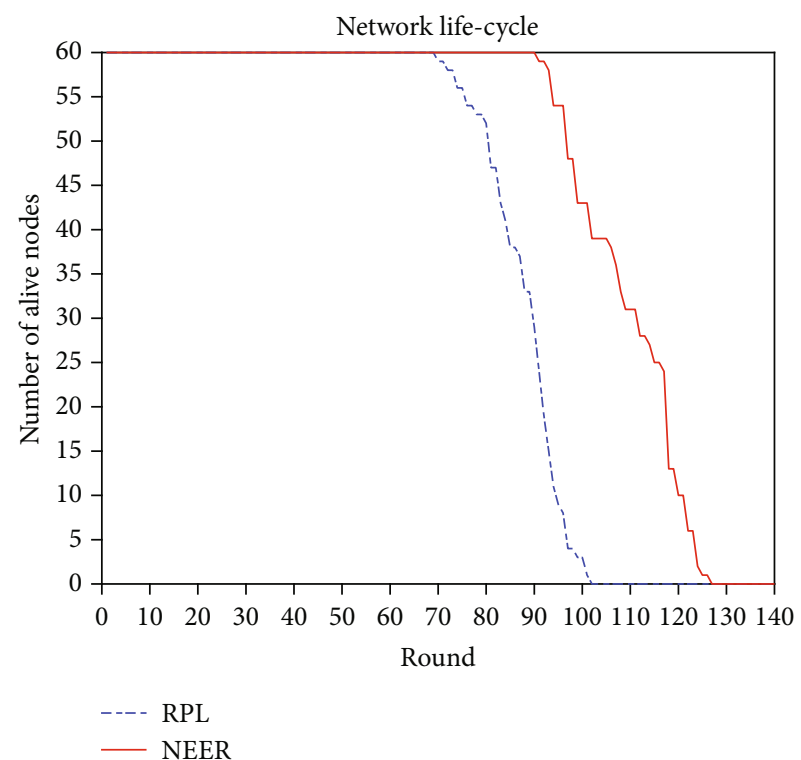

(c)

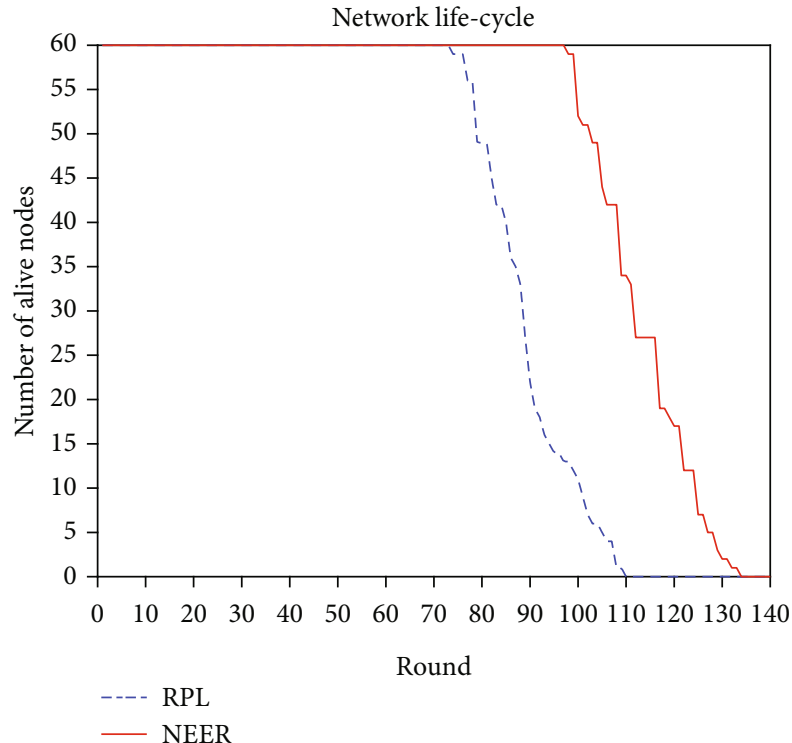

(b)

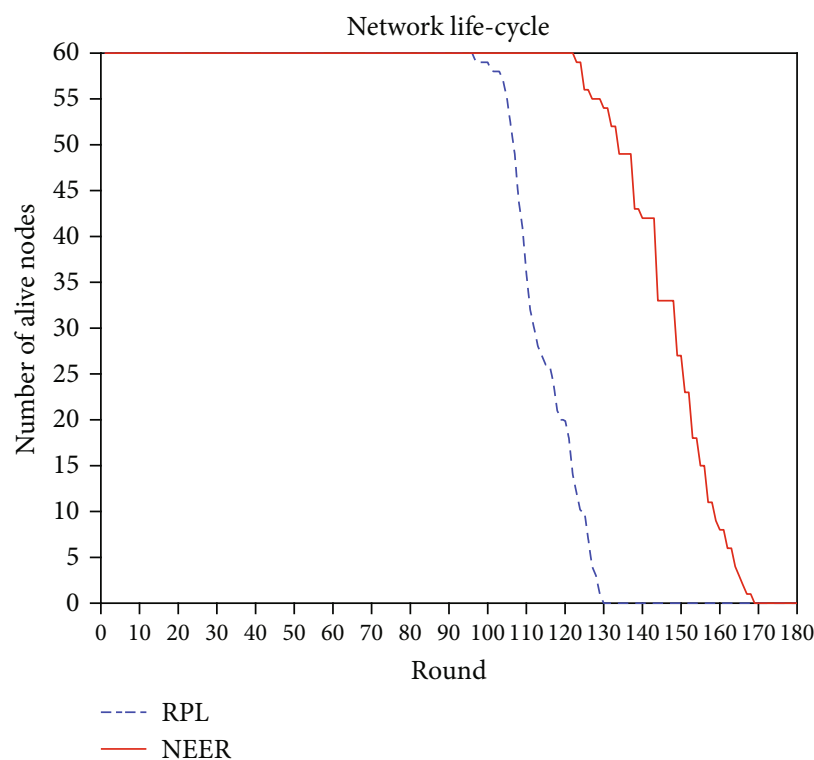

(d)

FIgURE 19: The network life cycle in multiple-edge area networks: (a) position 1 with three base stations; (b) position 2 with three base stations; (c) position 1 with four base stations; (d) position 2 with four base stations.

networks having only one base station and the number of sensor nodes in the WSN was constant, the more base stations were used, the more edge area networks were included in the WSN, and the smaller the number of sensor nodes per a single-edge area network was, thereby extending the life of the entire network and reducing the energy consumption. The results showed that the proposed NEER method had a longer network life and lower energy consumption of data transmission than the RPL protocol under the same number of base stations.

(2) Effect of Base Station Position. This experiment was performed to analyze the effect of the base station position on the NEER method's performance, and the proposed method's performance was compared with the RPL protocol's performance. The network topologies consisting of three base station nodes and 60 sensor nodes and four base station nodes and 60 sensor nodes randomly deployed in an area of $100 \mathrm{~m} \times 100 \mathrm{~m}$ were used in the experiment. Only the positions of base station nodes were changed in the experiment. In the experiments of three base stations and four base stations, each experiment has two kinds of base station position distribution, position 1 and position 2, where the distribution of the number of sensor nodes is relatively more balanced in each edge area network at position 2 than at position 1 . The upper limit of the transmission range of the sensor nodes was set to $20 \mathrm{~m}$, and the initial energy of the sensor nodes was $100 \mathrm{~mJ}$. The network 


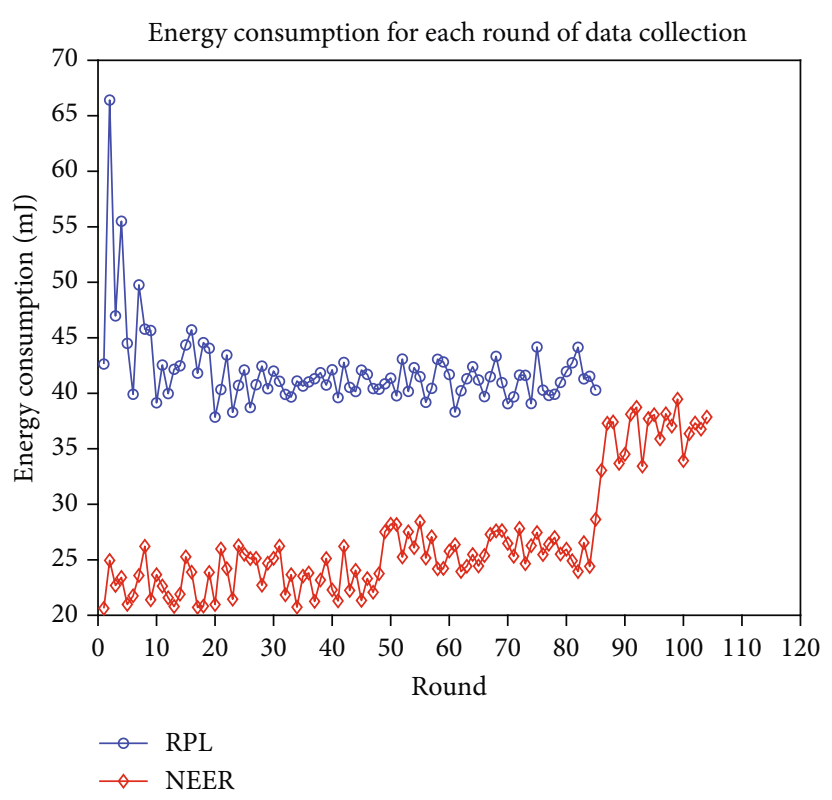

(a)

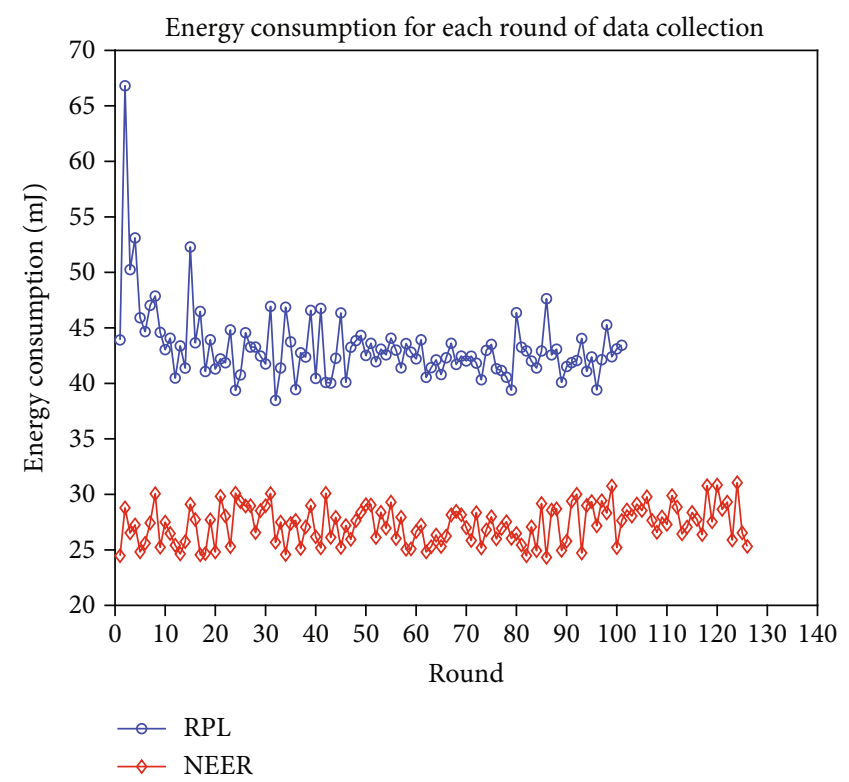

(c)

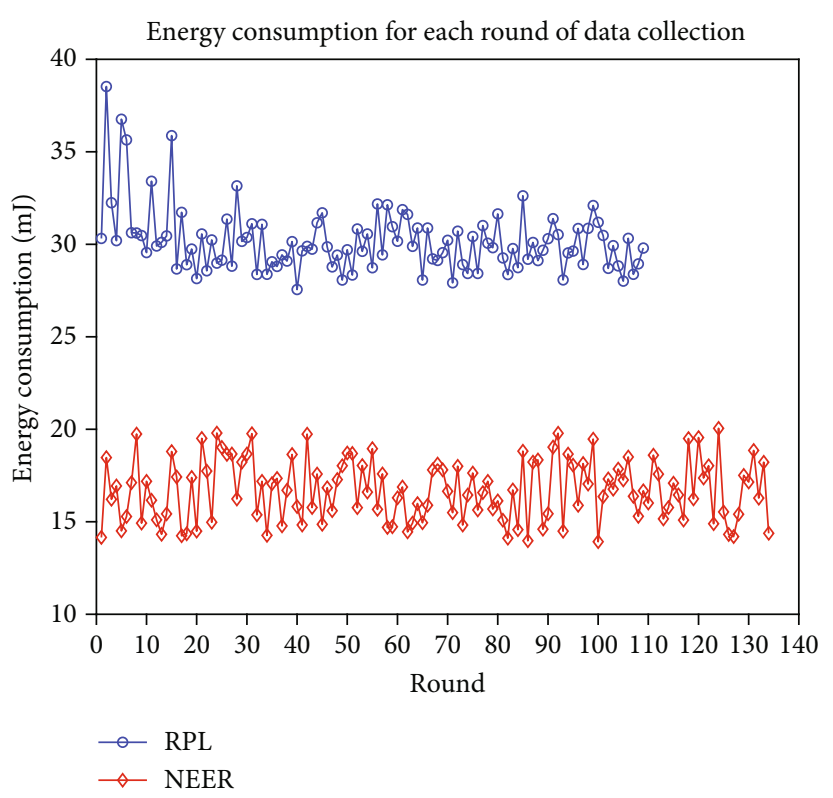

(b)

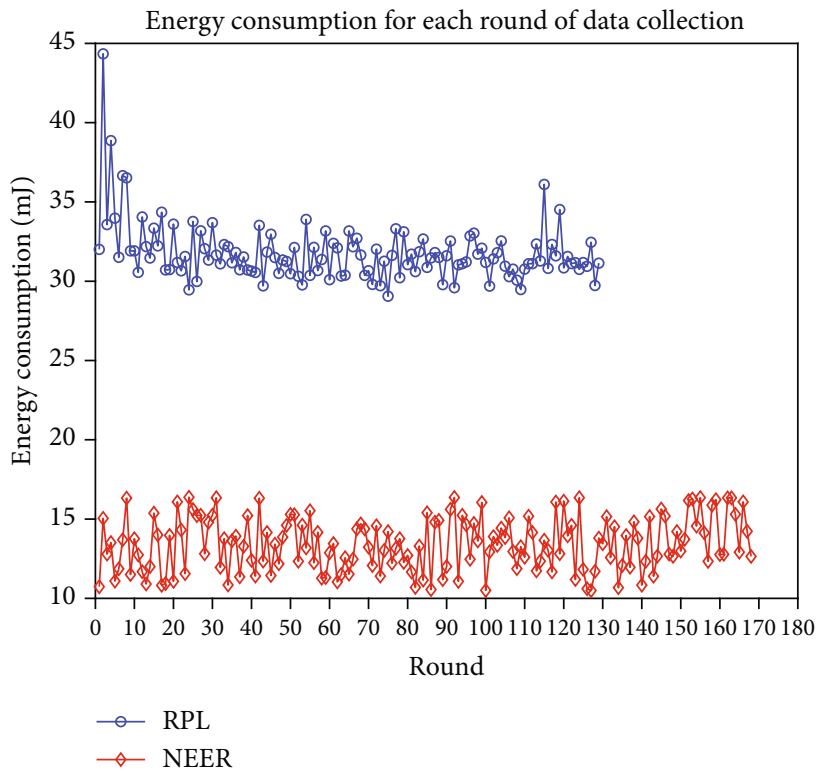

(d)

Figure 20: Comparison of energy consumption of data collection in multiple-edge area networks: (a) position 1 with three base stations; (b) position 2 with three base stations; (c) position 1 with four base stations; (d) position 2 with four base stations.

topologies of multiple-edge area networks with different positions of base stations generated by the RPL protocol and the proposed method are presented in Figures 17(a)17(d) and Figures 18(a)-18(d), respectively. In Figures 17 and 18 , red dots represent base stations (or gateways).

The comparison of network life and energy consumption between the RPL and NEER routing methods for network topologies consisting of three and four base stations at different positions is presented in Figures 19(a)-19(d) and Figures 20(a)-20(d), respectively. As presented, under other conditions unchanged, the network life of Figure 19(b) is longer than that of Figure 19(a), and the network energy consumption of Figure 20(b) is lower than that of Figure 20(a).
Similarly, the network life of Figure 19(d) is longer than that of Figure 19(c), and the network energy consumption of Figure $20(d)$ is lower than that of Figure 20(c). In addition, under the same number of base stations, the difference in the number of sensor nodes between the edge area networks having the base stations at position 2 is small, while at position 1 , the difference is significant, making the distribution of nodes unbalanced and resulting in unbalanced energy consumption of the network, thereby reducing the overall life of the network. Finally, the NEER method had lower energy consumption and a longer lifetime than the RPL protocol.

In order to present the effects of the two methods more intuitively, the experimental results of the NEER and RPL 
TABLE 6: Comparison of experimental results of NEER and RPL.

\begin{tabular}{|c|c|c|c|c|c|c|c|c|c|c|}
\hline & \multicolumn{3}{|c|}{ RPL } & \multicolumn{3}{|c|}{ NEER } & \multirow{2}{*}{ PLIF } & \multirow{2}{*}{ PLIA } & \multirow{2}{*}{ PECD } \\
\hline & & FND & AND & AEC & FND & AND & AEC & & & \\
\hline \multirow{4}{*}{ Number of sensor nodes } & 20 & 64 & 105 & 11.8 & 95 & 143 & 6.79 & $48 \%$ & $36 \%$ & $42 \%$ \\
\hline & 40 & 43 & 81 & 25.7 & 56 & 116 & 15.82 & $30 \%$ & $43 \%$ & $38 \%$ \\
\hline & 60 & 28 & 69 & 41.66 & 37 & 88 & 25.17 & $32 \%$ & $28 \%$ & $40 \%$ \\
\hline & 80 & 19 & 51 & 60.84 & 25 & 68 & 34.72 & $32 \%$ & $33 \%$ & $43 \%$ \\
\hline \multirow{4}{*}{ Network area size } & $100 \mathrm{~m} \times 100 \mathrm{~m}$ & 43 & 81 & 25.7 & 56 & 116 & 15.82 & $30 \%$ & $43 \%$ & $38 \%$ \\
\hline & $200 \mathrm{~m} \times 200 \mathrm{~m}$ & 22 & 40 & 60.75 & 30 & 52 & 37.99 & $36 \%$ & $30 \%$ & $37 \%$ \\
\hline & $400 \mathrm{~m} \times 400 \mathrm{~m}$ & 11 & 21 & 109.18 & 15 & 27 & 66.62 & $36 \%$ & $29 \%$ & $39 \%$ \\
\hline & $800 \mathrm{~m} \times 800 \mathrm{~m}$ & 6 & 14 & 167.1 & 9 & 23 & 150.48 & $50 \%$ & $64 \%$ & $10 \%$ \\
\hline \multirow{4}{*}{ Distribution of initial energy } & Uniform distribution & 43 & 79 & - & 54 & 115 & - & $27 \%$ & $47 \%$ & - \\
\hline & Normal distribution & 41 & 87 & - & 57 & 122 & - & $38 \%$ & $40 \%$ & - \\
\hline & Binomial distribution & 43 & 81 & - & 56 & 114 & - & $31 \%$ & $42 \%$ & - \\
\hline & Poisson distribution & 43 & 81 & - & 52 & 115 & - & $20 \%$ & $43 \%$ & - \\
\hline \multirow{4}{*}{ Number of base stations } & 3 & 55 & 91 & 34.86 & 70 & 118 & 21.11 & $27 \%$ & $30 \%$ & $39 \%$ \\
\hline & 4 & 78 & 117 & 34.08 & 104 & 151 & 18.67 & $33 \%$ & $29 \%$ & $45 \%$ \\
\hline & 5 & 105 & 138 & 31.41 & 128 & 173 & 17.54 & $22 \%$ & $25 \%$ & $44 \%$ \\
\hline & 6 & 129 & 172 & 30.6 & 156 & 224 & 17.14 & $21 \%$ & $30 \%$ & $44 \%$ \\
\hline \multirow{4}{*}{ Position of base stations } & Position 1 of three base stations & 34 & 86 & 40.02 & 48 & 105 & 29.84 & $41 \%$ & $22 \%$ & $25 \%$ \\
\hline & Position 2 of three base stations & 74 & 110 & 30.18 & 98 & 134 & 19.88 & $32 \%$ & $22 \%$ & $34 \%$ \\
\hline & Position 1 of four base stations & 70 & 102 & 43.28 & 91 & 127 & 30.43 & $30 \%$ & $25 \%$ & $30 \%$ \\
\hline & Position 2 of four base stations & 97 & 130 & 31.78 & 123 & 169 & 16.43 & $27 \%$ & $30 \%$ & $48 \%$ \\
\hline \multicolumn{2}{|c|}{ Average percentage of decrease or increase } & & & & & & & $32 \%$ & $35 \%$ & $37 \%$ \\
\hline
\end{tabular}

FND: first node died; AND: all nodes died; AEC: average energy consumption; PLIF: percentage of life cycle increase according to the first node died; PLIA: percentage of life cycle increase according to all nodes died; PECD: percentage of energy consumption decrease.

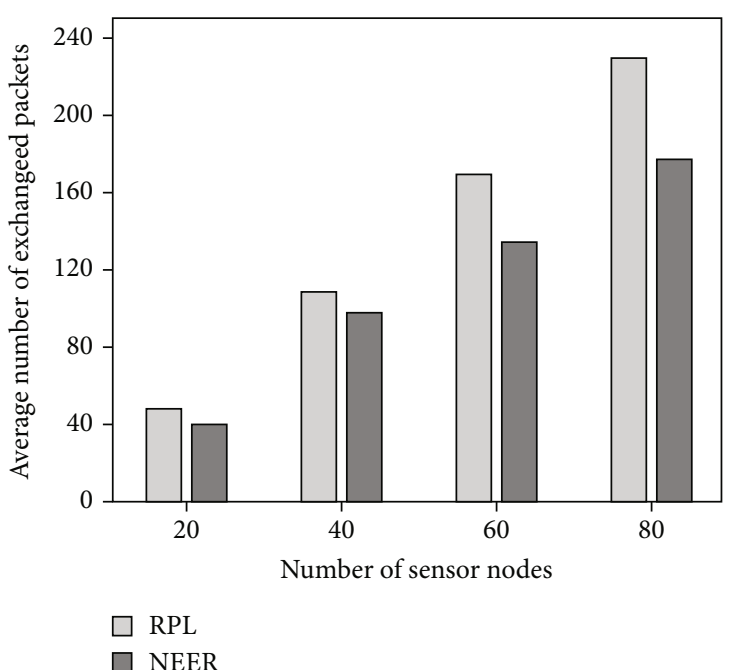

(a)

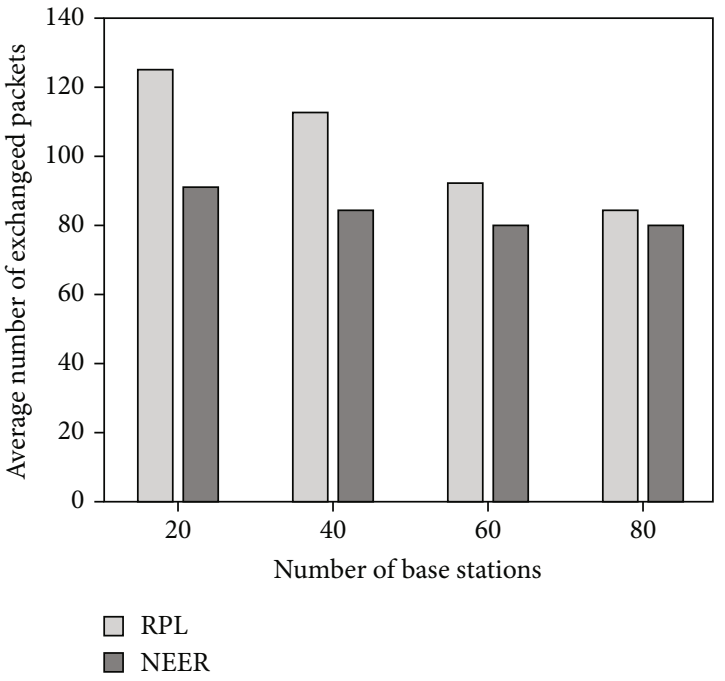

(b)

Figure 21: Comparison of the average number of exchanged packets in data collection: (a) different numbers of sensor nodes in the singleedge area network; (b) different numbers of base stations in the multiedge area network.

are listed in Table 6. On average, compared with the RPL, the NEER method extended the network lifetime by at least $30 \%$ and reduced the energy consumption by approximately $37 \%$.
5.2.3. Communication Cost Analysis. The communication cost was analyzed under different numbers of sensor nodes and base stations. The comparison of the number of 
exchanged packets per round between the RPL and NEER routing methods is presented in Figures 21(a) and 21(b). In Figure 21(a), the case of a single-edge area network is presented; meanwhile, in Figure 21(b), the case of a multiedge area network is displayed. Under other conditions unchanged, as shown in Figure 21(a), the larger the number of sensor nodes was, the larger the number of exchanged packets was. Additionally, as shown in Figure 21(b), the larger the number of base stations was, the smaller the number of exchanged packets was. In both considered cases, the number of exchanged packets of the NEER method was smaller than that of the RPL method. This was mainly because the NEER method collected data of all nodes on the path by one-time routing, so the packet exchange rate was low, and the communication overhead was reduced.

\section{Conclusions}

This paper proposes a novel energy-efficient, static scenariooriented routing method of WSNs based on the edge computing method, named the NEER, which is applicable to the scenario with fixed positions of sensor nodes. Based on the idea of edge computing, a WSN is divided into several edge area networks according to the base station coverage. The proposed method is a semicentralized, energy-efficient WSN routing method. The proposed method includes three parts: topology discovery, route calculation, and data transmission. For a single-edge area network, based on a weighted undirected graph model and combined with the residual energy of the sensor node, a base station calculates an optimal energy consumption path for each of the sensor nodes, while the sensor nodes perform only data transmission along the optimal paths determined by the base station. Since data of a single-edge area network is routed with the minimum energy consumption, the data transmission of the entire WSN achieves the lowest energy consumption.

The proposed NEER method is verified using the Contiki/Cooja platform from the aspects of two indicators, the network life cycle and energy consumption, and it was compared with the RPL protocol from the perspectives of the number of sensor nodes, network scale, initial energy distribution, the number of base stations, and base station locations. The experimental results show that the NEER method can extend the WSN lifetime by at least 30\% and reduce the energy consumption by about $37 \%$ compared with the RPL. Under the same conditions, the number of exchanged packets of the NEER method is lower than that of the RPL method.

In addition, the experimental results also show that the more nodes in the sensor network are, the larger the network scale, the greater the energy consumption of the network, and the shorter the network lifetime will be. Although increasing the number of base stations can reduce network energy consumption and extend network life, blindly increasing the base station number will cause a problem in sensor node coverage effectiveness and cost growth. At the same time, based on the results, the base station location affects the lifetime and energy consumption of a network.
Therefore, when deploying base stations, the number of nodes covered by each base station should be balanced.

Although the proposed method can achieve good routing performance, future research is necessary. Namely, it should be studied how to determine a base station's location to maximize the usage of sensor node resources. Additionally, it should be examined how to redivide the edge area network and reconstruct the network topology when the network topology updates. Lastly, adaptive routing protocol should be verified in general scenarios.

\section{Data Availability}

No data were used to support this study.

\section{Conflicts of Interest}

The authors declare that they have no conflicts of interest.

\section{Acknowledgments}

This research was supported in part by the NSFC (National Natural Science Foundation of China) program, under Grant Nos. 61672372, 61472211, and 61472268; Natural Science Fund for Colleges and Universities in Jiangsu Province under Grant No. 19KJB520056; Science and Technology Entrepreneurship Angel Program of Suzhou under Grant CYTS2019255; Science and Technology Project of Suzhou under Grant SS202151; Jiangsu Universities Overseas Research Program in 2019, the Outstanding ScienceTechnology Innovation Team Program of Colleges and Universities in Jiangsu; the Industrial Technology Innovation Project of Suzhou City under Grant No. SYG201710; and the Program to Cultivate Middle-aged and Young Cadre Teacher of Suzhou Vocational University.

\section{References}

[1] J. Yang, M. Ding, G. Mao, Z. Lin, D. G. Zhang, and T. H. Luan, "Optimal base station antenna downtilt in downlink cellular networks," IEEE Transactions on Wireless Communications, vol. 18, no. 3, pp. 1779-1791, 2019.

[2] W. Z. Khan, E. Ahmed, S. Hakak, I. Yaqoob, and A. Ahmed, "Edge computing: a survey," Future Generation Computer Systems, vol. 97, pp. 219-235, 2019.

[3] T. Qiu, N. Chen, K. Li, M. Atiquzzaman, and W. Zhao, "How can heterogeneous internet of things build our future: a survey," IEEE Communication Surveys and Tutorials, vol. 20, no. 3, pp. 2011-2027, 2018.

[4] B. Prabhu and S. Sophia, "Issues in environmental pollution monitoring using distributed wireless sensor network," Pollution Research, vol. 34, pp. 199-205, 2015.

[5] T. Qiu, K. Zheng, M. Han, C. L. P. Chen, and M. Xu, “A dataemergency-aware scheduling scheme for Internet of Things in smart cities," IEEE Transactions on Industrial Informatics, vol. 14, no. 5, pp. 2042-2051, 2018.

[6] A. Mathur, T. Newe, and M. Rao, "Defence against black hole and selective forwarding attacks for medical WSNs in the IoT," Sensors, vol. 16, no. 1, p. 118, 2016. 
[7] T. Qiu, R. Qiao, and D. O. Wu, "EABS: an event-aware backpressure scheduling scheme for emergency Internet of Things," IEEE Transactions on Mobile Computing, vol. 17, no. 1, pp. 72-84, 2018.

[8] J. Chen, G. Mao, C. Li, W. Liang, and D.-g. Zhang, "Capacity of cooperative vehicular networks with infrastructure support: multiuser case," IEEE Transactions on Vehicular Technology, vol. 67, no. 2, pp. 1546-1560, 2017.

[9] J. A. Jiang, C. H. Wang, C. H. Chen et al., "A WSN-based automatic monitoring system for the foraging behavior of honey bees and environmental factors of beehives," Computers and Electronics in Agriculture, vol. 123, pp. 304-318, 2016.

[10] T. Winter, P. Thubert, A. Brandt et al., "RPL: IPv6 routing protocol for low-power and lossy networks," RFC, vol. 6550, pp. 1-157, 2012.

[11] R. Wenling and P. Zeng, Study and performance analysis of $R P L$ routing protocol in WSN, Microcomputer \& Its Applications, 2017.

[12] H. Song, Z. Xu, and X. Wang, "Energy-efficient flooding with minimum latency for low-duty-cycle WSNs," in 2016 10th International Conference on Sensing Technology (ICST), pp. 1-6, Nanjing, China, 2016.

[13] A. Razaque, S. Mudigulam, K. Gavini, F. Amsaad, M. Abdulgader, and G. S. Krishna, "H-LEACH: hybrid-low energy adaptive clustering hierarchy for wireless sensor networks," in 2016 IEEE Long Island Systems, Applications and Technology Conference (LISAT), pp. 1-4, Farmingdale, NY, USA, 2016.

[14] W. Yanqin, "Analysis and improvement of GEAR routing protocol," Microcontrollers \& Embedded Systems, p. 08, 2011.

[15] R. A. Uthra and S. K. Raja, "QoS routing in wireless sensor networks-a survey," ACM Computing Surveys, vol. 45, no. 1, pp. 1-12, 2012.

[16] D. Kandris, G. Tselikis, E. Anastasiadis, E. Panaousis, and T. Dagiuklas, "COALA: a protocol for the avoidance and alleviation of congestion in wireless sensor networks," Sensors, vol. 17, no. 11, p. 2502, 2017.

[17] H. S. Z. Kazmi, N. Javaid, M. Awais, M. Tahir, S. O. Shim, and Y. B. Zikria, "Congestion avoidance and fault detection in WSNs using data science techniques," Transactions on Emerging Telecommunications Technologies, no. article e3756, 2019.

[18] M. Gholipour, A. Haghighat, and M. R. Meybodi, "Congestion avoidance in cognitive wireless sensor networks using TOPSIS and response surface methodology," Telecommunication Systems, vol. 67, no. 3, pp. 519-537, 2018.

[19] A. Ghaffari, "Congestion control mechanisms in wireless sensor networks: a survey," Journal of Network and Computer Applications, vol. 52, pp. 101-115, 2015.

[20] M. A. Jan, S. R. U. Jan, M. Alam, A. Akhunzada, and I. U. Rahman, "A comprehensive analysis of congestion control protocols in wireless sensor networks," Mobile Networks and Applications, vol. 23, no. 3, pp. 456-468, 2018.

[21] A. Bohloulzadeh and M. Rajaei, "A survey on congestion control protocols in wireless sensor networks," International Journal of Wireless Information Networks, vol. 27, no. 3, pp. 365-384, 2020.

[22] A. Rani and S. Kumar, "A survey of security in wireless sensor networks," in 2017 3rd International Conference on Computational Intelligence \& Communication Technology (CICT), pp. 1-5, Ghaziabad, India, 2017.

[23] I. Tomić and J. A. McCann, "A survey of potential security issues in existing wireless sensor network protocols," IEEE
Internet of Things Journal, vol. 4, no. 6, pp. 1910-1923, 2017.

[24] H. Xie, Z. Yan, Z. Yao, and M. Atiquzzaman, "Data collection for security measurement in wireless sensor networks: a survey," IEEE Internet of Things Journal, vol. 6, no. 2, pp. 22052224, 2019.

[25] R. Kumar, S. Tripathi, and R. Agrawal, “An analysis and comparison of security protocols on wireless sensor networks (WSN)," in Design Frameworks for Wireless Networks, pp. 321, Springer, 2020.

[26] M. S. Ibrahem, M. Nazri, and Z. Othman, "Wireless sensor networks: an overview of multi-objective optimization problems," Journal of Advance Research in Dynamical \& Control Systems, vol. 10, pp. 1569-1588, 2018.

[27] Z. Fei, B. Li, S. Yang, C. Xing, H. Chen, and L. Hanzo, "A survey of multi-objective optimization in wireless sensor networks: metrics, algorithms, and open problems," IEEE Communication Surveys and Tutorials, vol. 19, no. 1, pp. 550-586, 2017.

[28] D. Kandris, A. Alexandridis, T. Dagiuklas, E. Panaousis, and D. D. Vergados, Multiobjective Optimization Algorithms for Wireless Sensor Networks, Hindawi, 2020.

[29] D. Praveen Kumar, T. Amgoth, and C. S. R. Annavarapu, "Machine learning algorithms for wireless sensor networks: a survey," Information Fusion, vol. 49, pp. 1-25, 2019.

[30] S. Lata and S. Mehfuz, "Machine learning based energy efficient wireless sensor network," in 2019 International Conference on Power Electronics, Control and Automation (ICPECA), pp. 1-5, New Delhi, India, 2019.

[31] F. Zantalis, G. Koulouras, S. Karabetsos, and D. Kandris, "A review of machine learning and IoT in smart transportation," Future Internet, vol. 11, no. 4, p. 94, 2019.

[32] R. Mittal and M. S. Bhatia, "Wireless sensor networks for monitoring the environmental activities," in IEEE International Conference on Computational Intelligence \& Computing Research, Coimbatore, India, 2011.

[33] Y. Zhu, J. Song, and F. Dong, "Applications of wireless sensor network in the agriculture environment monitoring," Procedia Engineering, vol. 16, pp. 608-614, 2011.

[34] J. Aponte-Luis, J. A. Gómez-Galán, F. Gómez-Bravo, M. Sánchez-Raya, J. Alcina-Espigado, and P. Teixido-Rovira, "An efficient wireless sensor network for industrial monitoring and control," Sensors, vol. 18, no. 2, p. 182, 2018.

[35] N. Sghaier, A. Mellouk, B. Augustin et al., "Wireless sensor networks for medical care services," in 2011 7th International Wireless Communications and Mobile Computing Conference, pp. 571-576, Istanbul, Turkey, 2011.

[36] S. K. Gharghan, S. L. Mohammed, A. al-Naji et al., “Accurate fall detection and localization for elderly people based on neural network and energy-efficient wireless sensor Network," Energies, vol. 11, no. 11, p. 2866, 2018.

[37] I. Khan, F. Belqasmi, R. Glitho, N. Crespi, M. Morrow, and P. Polakos, "Wireless sensor network virtualization: a survey," IEEE Communication Surveys and Tutorials, vol. 18, no. 1, pp. 553-576, 2016.

[38] A. Velinov and A. Mileva, "Running and testing applications for Contiki OS using Cooja simulator," in International Conference on Information Technology and Development of Education - ITRO 2016, Zrenjanin, Republic of Serbia, 2016.

[39] B. Bagula and Z. Erasmus, "Iot emulation with Cooja," in ICTP-IoT workshop, Trieste, 2015. 